\title{
THE TUTTE POLYNOMIAL OF THE SIERPIŃSKI AND HANOI GRAPHS
}

\author{
ALFREDO DONNO \\ Dipartimento di Matematica, Sapienza Università di Roma, Piazzale A. Moro, 2 \\ 00185 Roma, Italia. E-mail address: alfredo.donno@sbai.uniroma1.it \\ DONATELLA IACONO \\ Dipartimento di Matematica, Università degli Studi di Bari, Via E. Orabona, 4 \\ 70125 Bari, Italia. E-mail address: iacono@dm.uniba.it
}

\begin{abstract}
We study the Tutte polynomial of two infinite families of finite graphs: the Sierpiński graphs, which are finite approximations of the well-known Sierpiński gasket, and the Schreier graphs of the Hanoi Towers group $H^{(3)}$ acting on the rooted ternary tree. For both of them, we recursively describe the Tutte polynomial and we compute several special evaluations of it, giving interesting results about the combinatorial structure of these graphs.
\end{abstract}

\section{INTRODUCTION}

The Tutte polynomial is a two-variable polynomial which can be associated with a graph, a matrix, or, more generally, with a matroid. It has many interesting applications in several areas of sciences as, for instance, Combinatorics, Probability, Statistical Mechanics, Computer Science and Biology. It was introduced by W.T. Tutte [28, 29, 30] and we will mainly refer to [5, 6, 16, 31] as expository papers.

Given a finite graph $G$, its Tutte polynomial $T(G ; x, y)$ satisfies a fundamental universal property with respect to the deletion-contraction reduction of the graph. Hence, any multiplicative graph invariant with respect to a deletion-contraction reduction turns out to be an evaluation of it. This polynomial is quite interesting since several combinatorial, enumerative and algebraic properties of the graph can be investigated by considering special evaluations of it. For instance, one gets information about the number of spanning trees, spanning connected subgraphs, spanning forests and acyclic orientations of the graph. Moreover, the Tutte polynomial also allows to recover the reliability and the chromatic polynomials. It has also many interesting connections with statistical mechanical models as the Potts model [32, the percolation [25], the Abelian Sandpile Model [12, 22], as well as with the theory of error correcting codes [32].

In this paper, we study the Tutte polynomial of two infinite families of finite graphs very close each other: the Sierpiński graphs $\left\{\Gamma_{n}\right\}_{n \geq 1}$, approximating the famous Sierpiński gasket, and the Schreier graphs $\left\{\Sigma_{n}\right\}_{n \geq 1}$ of the Hanoi Towers group $H^{(3)}$, whose action on the ternary tree models the well-known Hanoi Towers game on three pegs, and which is an example of selfsimilar group. In the last decades, the study of automorphism groups of rooted trees has been largely investigated: R. Grigorchuk and a number of coauthors have developed a new exciting direction of research focusing on finitely generated groups acting by automorphisms on rooted trees [2]. They proved that these groups have deep connections with the theory of profinite

Mathematics Subject Classification (2010): Primary: 05C31. Secondary: 05C15, 05C30, 05C38, $20 \mathrm{E} 08$.

Key words and phrases. Tutte polynomial, Sierpiński graph, Schreier graph of the Hanoi Towers group, selfsimilar graph, spanning subgraph, acyclic orientation, reliability polynomial, chromatic polynomial, partition function of the Ising model. 
groups and with complex dynamics. In particular, many groups of this type satisfy a property of self-similarity, reflected on fractalness of some limit objects associated with them [23]. In [7], the Tutte polynomial is studied for two examples of Schreier graphs associated with the action of two self-similar groups on the rooted binary tree, namely the Grigorchuk group and the Basilica group.

In this paper, we follow a combinatorial approach and we use the self-similar structure of the graphs (in the sense of [27]) to recursively investigate the Tutte polynomial of the families $\left\{\Gamma_{n}\right\}_{n \geq 1}$ and $\left\{\Sigma_{n}\right\}_{n \geq 1}$. It is worth mentioning [4, 24, where the authors consider the Tutte polynomial for recursive families of graphs.

We study the Tutte polynomial analyzing all the spanning subgraphs of these sequences of graphs. Then, we give a partition of the set of the spanning subgraphs, that allows us to split the polynomial as a sum of three terms (the same strategy turns out to be a powerful tool to investigate many combinatorial and statistical models on them: see, for instance, [8, 9, 10, 11, 13, 14, 15, 26, 27]). Finally, using self-similarity, we are able to give a recursive formula for each of these terms (see Theorems 3.1 and 4.31). Once we have these formulas, we are able to show the following properties:

- recursive formulas for the Tutte polynomial (Theorems 3.1 and 4.3);

- description of the reliability polynomial (Propositions 3.3 and 4.7);

- computation of complexity (Propositions 3.5 and 4.8);

- number of connected spanning subgraphs (Propositions 3.7 and 4.10);

- number of spanning forests (Propositions 3.9 and 4.11);

- number of acyclic orientations (Propositions 3.12 and 4.13);

- description of the chromatic polynomial (Propositions 3.14 and 4.14);

- computation of the partition function of the Ising model (Theorems 3.17 and 4.15).

Some of these combinatorial properties were already known in literature, and so our main result, which consists in the description of the Tutte polynomial, collects these aspects in a more general and stronger context.

The paper is organized as follows. Section 2 contains some preliminary material on the Tutte polynomial and on the theory of automorphism groups of rooted regular trees. In Section 3, we study the Tutte polynomial of the Sierpiński graphs $\left\{\Gamma_{n}\right\}_{n \geq 1}$ and analyze many applications. In particular, we recover that, for every $n \geq 1$, the graph $\Gamma_{n}$ is uniquely 3 -colorable (Proposition 3.15). Section 4 is devoted to the analysis of the Schreier graphs $\left\{\Sigma_{n}\right\}_{n \geq 1}$ of the Hanoi Towers group $H^{(3)}$. We also underline the very close structure of the graphs $\left\{\Gamma_{n}\right\}_{n \geq 1}$ and $\left\{\Sigma_{n}\right\}_{n \geq 1}$ by pointing out the relationship between $T\left(\Gamma_{n} ; x, y\right)$ and $T\left(\Sigma_{n} ; x, y\right)$ (Proposition 4.4).

While submitting this paper, we became aware of a recent preprint [1, submitted to arXiv after our one, where similar computations are performed.

\section{Preliminaries}

2.1. The Tutte polynomial. Throughout the paper, we deal with graphs which are connected and finite. Moreover, both multiple edges and multiple loops are allowed. As usual, $G=$ $(V(G), E(G))$ denotes a graph with vertex set $V(G)$ and edge set $E(G)$; we will often write $V$ and $E$, when there is no risk of confusion, and so $G=(V, E)$. Moreover, we denote by $E_{n}$ the graph with $n$ vertices and no edges, and by $K_{n}$ the complete graph on $n$ vertices. A subgraph $A=(V(A), E(A))$ of a graph $G=(V(G), E(G))$ is said spanning if the condition $V(A)=V(G)$ 
is satisfied. In particular, a spanning subtree of $G$ is a spanning subgraph of $G$ which is a tree, a spanning forest of $G$ is a spanning subgraph of $G$ which is a forest. The number of spanning trees of a graph $G$ is called complexity of $G$ and is denoted by $\tau(G)$. It is interesting to study complexity when the system grows. More precisely, given a sequence $\left\{G_{n}\right\}_{n \geq 1}$ of finite graphs with complexity $\tau\left(G_{n}\right)$, such that $\left|V\left(G_{n}\right)\right| \rightarrow \infty$, the limit

$$
\lim _{\left|V\left(G_{n}\right)\right| \rightarrow \infty} \frac{\log \tau\left(G_{n}\right)}{\left|V\left(G_{n}\right)\right|},
$$

when it exists, is called the asymptotic growth constant of the spanning trees of $\left\{G_{n}\right\}_{n \geq 1}$ [21]. Finally, let $k(G)$ be the number of connected components of $G$.

Definition 2.1. Let $A$ be a spanning subgraph of $G$, then the rank $r(A)$ and the nullity $n(A)$ of $A$ are defined as

$r(A)=|V(A)|-k(A)=|V(G)|-k(A) \quad$ and $\quad n(A)=|E(A)|-r(A)=|E(A)|-|V(A)|+k(A)$.

Definition 2.2 (Spanning subgraphs). Let $G=(V, E)$ be a graph. The Tutte polynomial $T(G ; x, y)$ of $G$ is defined as

$$
T(G ; x, y)=\sum_{A \subseteq G}(x-1)^{r(G)-r(A)}(y-1)^{n(A)},
$$

where the sum runs over all the spanning subgraphs $A$ of $G$.

The Tutte polynomial can be also defined by a recursion process given by deleting and contracting edges. We recall that, given $G=(V, E)$, the graph $G \backslash e=(V, E-\{e\})$ is obtained from $G$ by deleting the edge $e \in E$. The graph obtained by contracting an edge $e \in E$ is the result of the identification of the endpoints of $e$ followed by removing $e$. We denote it by $G / e$. Finally, we recall that an edge in a connected graph is a bridge if its deletion disconnects the graph, it is a loop if its endpoints coincide.

Definition 2.3 (Deletion-Contraction). Let $G=(V, E)$ be a graph. The Tutte polynomial $T(G ; x, y)$ of $G$ is defined as

$$
T(G ; x, y)= \begin{cases}1 & \text { if } G=E_{1} ; \\ x T(G \backslash e ; x, y) & \text { if e is a bridge; } \\ y T(G \backslash e ; x, y) & \text { if e is a loop; } \\ T(G \backslash e ; x, y)+T(G / e ; x, y) & \text { if e is neither a bridge nor a loop. }\end{cases}
$$

The recursive process to compute the Tutte polynomial in this second definition is independent on the order in which the edges are chosen: this can be proven by showing that Definitions 2.2 and 2.3 are equivalent 6 .

Once we have the definition, we can state some of the main properties of the Tutte polynomial (for more details, see [5, 6, 16]). Recall that a one point join $G * H$ of two graphs $G$ and $H$ is obtained by identifying a vertex $v$ of $G$ and a vertex $w$ of $H$ into a single vertex of $G * H$. The following property can be easily proven by using Definition 2.2 .

$$
T(G * H ; x, y)=T(G ; x, y) T(H ; x, y) .
$$

In the sequel of the paper, we will refer to this equality as Property (2). Next, recall that if $G=(V, E)$ is connected and for some $W \subset V$ the graph $G \backslash W$ is disconnected, we say that $W$ separates $G$. A graph $G$ is 2-connected if either $G$ is the complete graph $K_{3}$ or it has at least 4 vertices and no vertex separates $G$. 
Theorem 2.4. [16, Theorem 26] If $G$ is a 2-connected graph, then $T(G ; x, y)$ is irreducible in $\mathbb{Z}[x, y]$.

It follows that, since the graphs $\Gamma_{n}$ and $\Sigma_{n}$ are 2-connected, for each $n \geq 1$, their Tutte polynomials are irreducible in $\mathbb{Z}[x, y]$.

In the next sections, we will be interested in special evaluations of the Tutte polynomial, that allow us to deduce many combinatorial and algebraic properties of the graphs considered. In the following theorem, we collect many of these properties that are well-known in literature.

Theorem 2.5. [16, Theorems 3 and 8] Let $G=(V, E)$ be a connected graph and denote by $T(G ; x, y)$ its Tutte polynomial. Then:

(1) $T(G ; 1,1)=\tau(G)$;

(2) $T(G ; 1,2)$ is the number of spanning connected subgraphs of $G$;

(3) $T(G ; 2,1)$ is the number of spanning forests of $G$;

(4) $T(G ; 2,2)=2^{|E|}$;

(5) $T(G ; 2,0)$ is the number of acyclic orientations of $G$, i.e., orientations having no oriented cycles.

Let $R(G, p)$ the reliability polynomial of the graph $G$. For a random model where each edge of $G$ is independently chosen to be active (or open) with probability $p$ or inactive (closed) with probability $1-p$, it provides the probability that there is a path of active edges between each pair of vertices of $G$. Next, let $\chi(G, \lambda)$ be the chromatic polynomial of $G$, giving, for all values $\lambda$, the number of proper $\lambda$-colorings of $G$. Finally, let $Z$ be the partition function of the Ising model, which is obtained as a special case of the $Q$-Potts model on $G$, for $Q=2$.

The connection with the Tutte polynomial is given by the following theorem.

Theorem 2.6. [16, 32] Let $G=(V, E)$ be a graph. Then,

(1) $R(G, p)=p^{|V(G)|-1}(1-p)^{|E(G)|-|V(G)|+1} T\left(G ; 1, \frac{1}{1-p}\right)$;

(2) $\chi(G, \lambda)=(-1)^{r(G)} \lambda^{k(G)} T(G ; 1-\lambda, 0)$;

(3) $Z=2\left(e^{2 \beta J}-1\right)^{|V(G)|-1} e^{-\beta J|E(G)|} T\left(G ; \frac{e^{2 \beta J}+1}{e^{2 \beta J}-1}, e^{2 \beta J}\right)$, where $J$ is a positive constant and $\beta$ is the "inverse temperature".

2.2. Groups of automorphisms of rooted regular trees. Let $T_{q}$ be the infinite regular rooted tree of degree $q$, i.e., the rooted tree in which each vertex has $q$ children. Each vertex of the $n$-th level of the tree can be regarded as a word of length $n$ in the alphabet $X=\{0,1, \ldots, q-1\}$. Moreover, one can identify the set $X^{\omega}$ of infinite words in $X$ with the set $\partial T_{q}$ of infinite geodesic rays starting at the root of $T_{q}$. Next, let $S<A u t\left(T_{q}\right)$ be a group acting on $T_{q}$ by automorphisms generated by a finite symmetric set of generators $Y$. Moreover, suppose that the action is transitive on each level of the tree.

Definition 2.7. The $n$-th Schreier graph of the action of $S$ on $T_{q}$, with respect to the generating set $Y$, is a graph whose vertex set coincides with the set of vertices of the $n$-th level of the tree, and two vertices $u, v$ are adjacent if and only if there exists $s \in Y$ such that $s(u)=v$. If this is the case, the edge joining $u$ and $v$ is labelled by $s$. 
The vertices of this graph are labelled by words of length $n$ in $X$ and the edges are labelled by elements of $Y$. The Schreier graph is thus a regular graph of degree $|Y|$ with $q^{n}$ vertices, and it is connected, since the action of $S$ is level-transitive.

Definition 2.8. 23] A finitely generated group $S<A u t\left(T_{q}\right)$ is self-similar if, for all $g \in S, x \in$ $X$, there exist $h \in S, y \in X$ such that

$$
g(x w)=y h(w)
$$

for all finite words $w$ in the alphabet $X$.

Self-similarity implies that $S$ can be embedded into the wreath product $\operatorname{Sym}(q)$ \& $S$, where $\operatorname{Sym}(q)$ denotes the symmetric group on $q$ elements, so that any automorphism $g \in S$ can be represented as

$$
g=\tau\left(g_{0}, \ldots, g_{q-1}\right)
$$

where $\tau \in \operatorname{Sym}(q)$ describes the action of $g$ on the first level of $T_{q}$ and $g_{i} \in S, i=0, \ldots, q-1$, is the restriction of $g$ on the full subtree of $T_{q}$ rooted at the vertex $i$ of the first level of $T_{q}$ (observe that any such subtree is isomorphic to $T_{q}$ ). Hence, if $x \in X$ and $w$ is a finite word in $X$, we have

$$
g(x w)=\tau(x) g_{x}(w) .
$$

The class of self-similar groups contains many interesting examples of groups which have exotic properties: for instance, the first Grigorchuk group and the Basilica group. We recall here that the first Grigorchuk group was the first example of a group of intermediate growth (see [17] for a detailed account and further references). As regards the Basilica group, it was introduced by R. Grigorchuk and A. Żuk in [19] as a group generated by a three-state automaton; it is an example of an amenable group (see [3]) not belonging to the class of subexponentially amenable groups and it can be described as an iterated monodromy group [23].

In Section 4.1, we will describe the Schreier graphs $\left\{\Sigma_{n}\right\}_{n \geq 1}$ of the Hanoi Towers group $H^{(3)}$, using the self-similar representation of its generators. In Section 4.2, we will compute the Tutte polynomial of the graphs $\left\{\Sigma_{n}\right\}_{n \geq 1}$, using their self-similar structure.

\section{The Tutte polynomial of the Sierpiński graphs}

In this section we study the Tutte polynomial of a sequence of graphs $\left\{\Gamma_{n}\right\}_{n \geq 1}$, approximating the famous Sierpiński gasket. For each $n \geq 1$, the graph $\Gamma_{n}$ is very close to the Schreier graph $\Sigma_{n}$ of the group $H^{(3)}$ considered in the next section. More precisely, one can obtain $\Gamma_{n}$ from $\Sigma_{n}$ by removing loops and contracting, at each step, all the special edges of $\Sigma_{n}$, joining two different elementary triangles (see Section [4.1). The graph $\Gamma_{n}$ is also self-similar in the sense of [27], as can be seen in the following picture,


where the subgraphs $G_{1}, G_{2}, G_{3}$ of $\Gamma_{n}$ are isomorphic to $\Gamma_{n-1}$, and they are joint at the vertices $v_{1}, v_{2}$ and $v_{3}$, called special vertices of $\Gamma_{n}$. Note that $v_{i} \in G_{j}$, for each $i \neq j$. It is easy to prove by induction the following equalities:

$$
\left|V\left(\Gamma_{n}\right)\right|=\frac{3^{n}+3}{2} \quad\left|E\left(\Gamma_{n}\right)\right|=3^{n} .
$$


We want to compute the Tutte polynomial $T\left(\Gamma_{n} ; x, y\right)$ by using Definition 2.2. First of all, we define the following partition of the set of the spanning subgraphs of $\Gamma_{n}$ :

- $D_{2, n}$ denotes the set of spanning subgraphs of $\Gamma_{n}$, where the three outmost vertices belong to the same connected component;

- $D_{1, n}^{u}$ denotes the set of spanning subgraphs of $\Gamma_{n}$, where the leftmost and rightmost vertices belong to the same connected component, and the upmost one belongs to a different connected component. Similarly, by rotation, $D_{1, n}^{r}$ (respectively $D_{1, n}^{l}$ ) denotes the set of spanning subgraphs of $\Gamma_{n}$, where the rightmost (respectively leftmost) vertex is not in the same connected component containing the two other outmost vertices;

- $D_{0, n}$ denotes the set of spanning subgraphs of $\Gamma_{n}$, where the three outmost vertices belong to three different connected components.

To draw a subgraph of $\Gamma_{n}$ of the previous types, we will use the following notation.
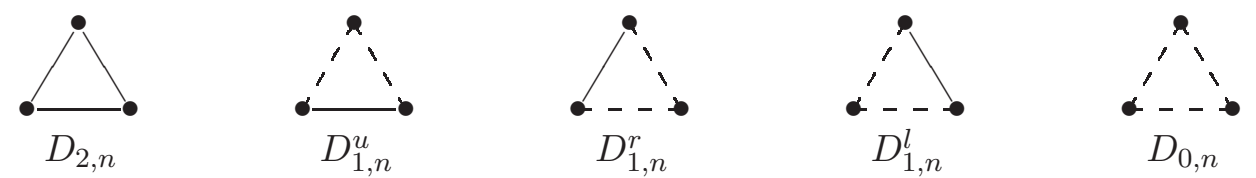

Hence, with our convention, a black line joining two outmost vertices in a diagram means that there is a path joining them in the subgraph and so they are in the same connected component. In the following pictures, we give explicit examples of spanning subgraphs of $\Gamma_{3}$, which are in $D_{2,3}, D_{1,3}^{u}$ and $D_{0,3}$, respectively.
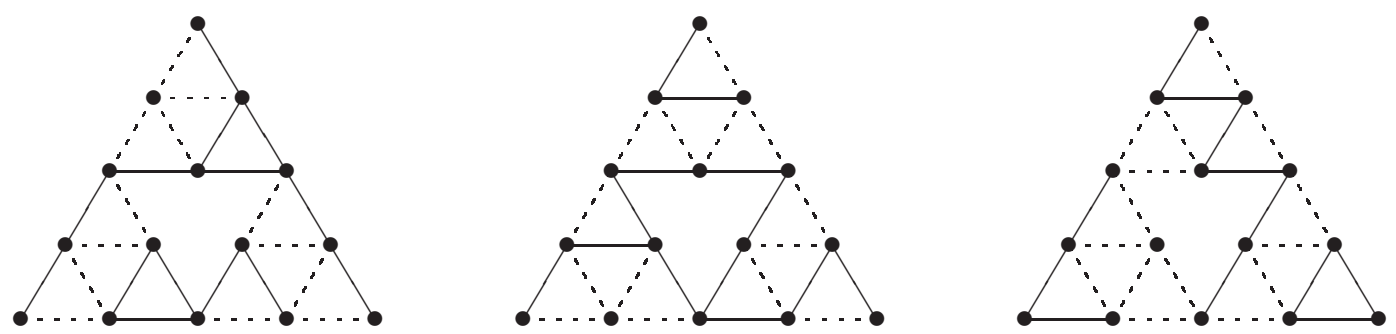

Observe that, for each $n \geq 1$, we have the partition

$$
D_{2, n} \sqcup D_{1, n}^{u} \sqcup D_{1, n}^{r} \sqcup D_{1, n}^{l} \sqcup D_{0, n}
$$

of the set of spanning subgraphs of $\Gamma_{n}$. Next, let us simply denote by $T_{n}(x, y)$ the Tutte polynomial $T\left(\Gamma_{n} ; x, y\right)$ of $\Gamma_{n}$ and define, for every $n \geq 1$, the following polynomials:

$$
\begin{aligned}
& \text { - } T_{2, n}(x, y)=\sum_{A \in D_{2, n}}(x-1)^{r\left(\Gamma_{n}\right)-r(A)}(y-1)^{n(A)} ; \\
& \text { - } T_{1, n}^{u}(x, y)=\sum_{A \in D_{1, n}^{u}}(x-1)^{r\left(\Gamma_{n}\right)-r(A)}(y-1)^{n(A)} ; \\
& \text { - } T_{0, n}(x, y)=\sum_{A \in D_{0, n}}(x-1)^{r\left(\Gamma_{n}\right)-r(A)}(y-1)^{n(A)} .
\end{aligned}
$$

Similarly, we define $T_{1, n}^{r}(x, y)$ and $T_{1, n}^{l}(x, y)$, by taking sums over $D_{1, n}^{r}$ and $D_{1, n}^{l}$, respectively. Note that, by the rotational-invariance of the graph $\Gamma_{n}$, one has

$$
T_{1, n}^{u}(x, y)=T_{1, n}^{r}(x, y)=T_{1, n}^{l}(x, y),
$$


so that we can simply use the notation $T_{1, n}(x, y)$ to denote one of these three polynomials. According with Definition 2.2 of the Tutte polynomial, we have:

$$
T_{n}(x, y)=T_{2, n}(x, y)+3 T_{1, n}(x, y)+T_{0, n}(x, y) .
$$

In order to give a recursive formula for $T_{n}(x, y)$, we provide recursive formulas for $T_{2, n}(x, y)$, $T_{1, n}(x, y)$ and $T_{0, n}(x, y)$ (Theorem 3.1). For this purpose, we have to analyze the relation between spanning subgraphs of $\Gamma_{n+1}$ and spanning subgraphs of $\Gamma_{n}$. The following key observation holds.

There exists a bijection between spanning subgraphs of $\Gamma_{n+1}$ and spanning subgraphs of the copies $G_{1}, G_{2}, G_{3}$ of $\Gamma_{n}$ inside $\Gamma_{n+1}$. This bijection is induced by restrictions.

Indeed, the restriction of a spanning subgraph $A$ of $\Gamma_{n+1}$ to the copies $G_{1}, G_{2}$ and $G_{3}$ of $\Gamma_{n}$ uniquely determines three spanning subgraphs $A_{1}, A_{2}$ and $A_{3}$ of $\Gamma_{n}$; viceversa, given three spanning subgraphs $A_{1}, A_{2}$ and $A_{3}$ of $G_{1}, G_{2}$ and $G_{3}$, respectively, then their union provides a spanning subgraph $A$ of the whole $\Gamma_{n+1}$.

Therefore, Equation (11) in Definition 2.2 for $\Gamma_{n+1}$ can be rewritten as

$$
T_{n+1}(x, y)=\sum_{A_{i} \subseteq G_{i}, i=1,2,3}(x-1)^{r\left(\Gamma_{n+1}\right)-r(A)}(y-1)^{n(A)},
$$

where $A_{i}$ is the restriction of $A$ to the subgraph $G_{i}$ of $\Gamma_{n+1}$.

Next, we need to understand how $r(A)$ and $n(A)$ depend on $r\left(A_{i}\right)$ and $n\left(A_{i}\right)$, for $i=1,2,3$. Firstly, observe that $r\left(\Gamma_{n+1}\right)=3 r\left(\Gamma_{n}\right)-1$ and $|V(A)|=\left|V\left(A_{1}\right)\right|+\left|V\left(A_{2}\right)\right|+\left|V\left(A_{3}\right)\right|-3$, for every spanning subgraph $A$ of $\Gamma_{n+1}$. Furthermore, two possibilities can occur.

If in the spanning subgraph $A$, obtained by the union of $A_{1}, A_{2}$ and $A_{3}$, the three special vertices are in the same connected component, and the two special vertices $v_{i}, v_{j} \in A_{k}$ are in the same connected component of $A_{k}$ for any $k=1,2,3$, then it is easy to check that

$$
k(A)=k\left(A_{1}\right)+k\left(A_{2}\right)+k\left(A_{3}\right)-2 \quad \text { and } \quad r(A)=r\left(A_{1}\right)+r\left(A_{2}\right)+r\left(A_{3}\right)-1 .
$$

Moreover, one has

$$
\begin{aligned}
n(A) & =\left(\left|E\left(A_{1}\right)\right|+\left|E\left(A_{2}\right)\right|+\left|E\left(A_{3}\right)\right|\right)-\left(\left|V\left(A_{1}\right)\right|+\left|V\left(A_{2}\right)\right|+\left|V\left(A_{3}\right)\right|-3\right) \\
& +\left(k\left(A_{1}\right)+k\left(A_{2}\right)+k\left(A_{3}\right)-2\right) \\
& =n\left(A_{1}\right)+n\left(A_{2}\right)+n\left(A_{3}\right)+1 .
\end{aligned}
$$

Hence, for such a spanning subgraph $A$ of $\Gamma_{n+1}$ (of "first type"), one gets:

$$
r\left(\Gamma_{n+1}\right)-r(A)=\sum_{i=1}^{3}\left(r\left(\Gamma_{n}\right)-r\left(A_{i}\right)\right) \quad \text { and } \quad n(A)=n\left(A_{1}\right)+n\left(A_{2}\right)+n\left(A_{3}\right)+1
$$

and so

$$
(x-1)^{r\left(\Gamma_{n+1}\right)-r(A)}(y-1)^{n(A)}=(y-1) \prod_{i=1}^{3}(x-1)^{r\left(\Gamma_{n}\right)-r\left(A_{i}\right)}(y-1)^{n\left(A_{i}\right)} .
$$

On the other hand, if in the spanning subgraph $A$ obtained by the union of $A_{1}, A_{2}$ and $A_{3}$ there are two special vertices $v_{i}, v_{j} \in A_{k}$ which do not belong to the same connected component of $A_{k}$, for some $k=1,2,3$ and $i, j \neq k$, it is easy to verify that

$$
k(A)=k\left(A_{1}\right)+k\left(A_{2}\right)+k\left(A_{3}\right)-3 \quad \text { and } \quad r(A)=r\left(A_{1}\right)+r\left(A_{2}\right)+r\left(A_{3}\right) .
$$


Moreover, one has in this case

$$
\begin{aligned}
n(A) & =\left(\left|E\left(A_{1}\right)\right|+\left|E\left(A_{2}\right)\right|+\left|E\left(A_{3}\right)\right|\right)-\left(\left|V\left(A_{1}\right)\right|+\left|V\left(A_{2}\right)\right|+\left|V\left(A_{3}\right)\right|-3\right) \\
& +\left(k\left(A_{1}\right)+k\left(A_{2}\right)+k\left(A_{3}\right)-3\right) \\
& =n\left(A_{1}\right)+n\left(A_{2}\right)+n\left(A_{3}\right) .
\end{aligned}
$$

Hence, for such a spanning subgraph $A$ of $\Gamma_{n+1}$ (of "second type"), one gets:

$$
r\left(\Gamma_{n+1}\right)-r(A)=\sum_{i=1}^{3}\left(r\left(\Gamma_{n}\right)-r\left(A_{i}\right)\right)-1 \quad \text { and } \quad n(A)=n\left(A_{1}\right)+n\left(A_{2}\right)+n\left(A_{3}\right)
$$

and so

$$
(x-1)^{r\left(\Gamma_{n+1}\right)-r(A)}(y-1)^{n(A)}=\frac{1}{(x-1)} \prod_{i=1}^{3}(x-1)^{r\left(\Gamma_{n}\right)-r\left(A_{i}\right)}(y-1)^{n\left(A_{i}\right)} .
$$

Theorem 3.1. For each $n \geq 1$, the Tutte polynomial $T_{n}(x, y)$ of $\Gamma_{n}$ is given by

$$
T_{n}(x, y)=T_{2, n}(x, y)+3 T_{1, n}(x, y)+T_{0, n}(x, y),
$$

where the polynomials $T_{2, n}(x, y), T_{1, n}(x, y), T_{0, n}(x, y) \in \mathbb{Z}[x, y]$ satisfy the following recursive relations:

$$
\begin{aligned}
T_{2, n+1}(x, y) & =(y-1) T_{2, n}^{3}+\frac{1}{x-1}\left(6 T_{2, n}^{2} T_{1, n}+3 T_{2, n} T_{1, n}^{2}\right) \\
T_{1, n+1}(x, y) & =(y-1) T_{2, n}^{2} T_{1, n}+\frac{1}{x-1}\left(T_{2, n}^{2} T_{0, n}+7 T_{2, n} T_{1, n}^{2}\right. \\
& \left.+2 T_{2, n} T_{1, n} T_{0, n}+4 T_{1, n}^{3}+T_{1, n}^{2} T_{0, n}\right) \\
T_{0, n+1}(x, y)= & (y-1)\left(3 T_{2, n} T_{1, n}^{2}+T_{1, n}^{3}\right)+\frac{1}{x-1}\left(12 T_{2, n} T_{1, n} T_{0, n}\right. \\
+ & \left.3 T_{2, n} T_{0, n}^{2}+14 T_{1, n}^{3}+24 T_{1, n}^{2} T_{0, n}+9 T_{1, n} T_{0, n}^{2}+T_{0, n}^{3}\right),
\end{aligned}
$$

with initial conditions

$$
T_{2,1}(x, y)=y+2 \quad T_{1,1}(x, y)=x-1 \quad T_{0,1}(x, y)=(x-1)^{2} .
$$

Proof. The initial conditions are easy to be verified. The strategy of the proof is to study all the possible choices of spanning subgraphs $A_{i}$ in the three copies $G_{i}$ of $\Gamma_{n}$ inside $\Gamma_{n+1}$, for $i=1,2,3$, and analyze which kind of contribution they give to $T_{2, n+1}(x, y), T_{1, n+1}(x, y)$ and $T_{0, n+1}(x, y)$.

Let us start by studying which configurations of spanning subgraphs $A_{i}$, for $i=1,2,3$, give a contribution to $T_{2, n+1}(x, y)$. First, we have the following configuration.

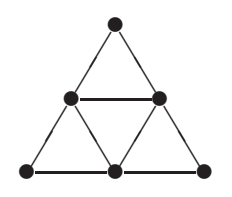

Here, we choose each $A_{i}$ in $D_{2, n}$; this contributes by a term $(y-1) T_{2, n}^{3}$, since in this subgraph the three special vertices are connected (first type). Moreover, we have two other possibilities, concerning spanning subgraphs of the second type, represented in the following two pictures. 

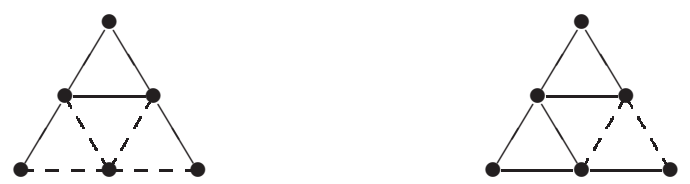

The first configuration contributes by a term $\frac{3}{x-1} T_{2, n} T_{1, n}^{2}$ (we have to consider the possible rotations); the second one contributes by $\frac{6}{x-1} T_{2, n}^{2} T_{1, n}$ (we have to take into account all the possible symmetries of $\left.\Gamma_{n+1}\right)$. This gives Equation (3).

Next, we study the contributions to the polynomial $T_{1, n+1}(x, y)$. The only case of subgraph of the first type is represented in the following picture.

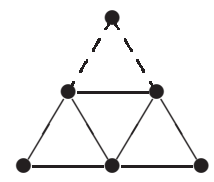

It gives a contribution of $(y-1) T_{2, n}^{2} T_{1, n}$. Then, consider the following configurations.
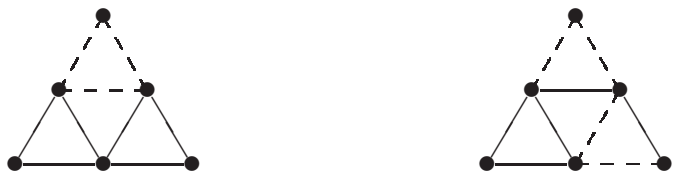

The first one contributes by $\frac{1}{x-1} T_{2, n}^{2} T_{0, n}$; the second one gives the contribution $\frac{2}{x-1} T_{2, n} T_{1, n}^{2}$ (we have to take into account one reflection with respect to the vertical axis). Let us analyze now the three following configurations.
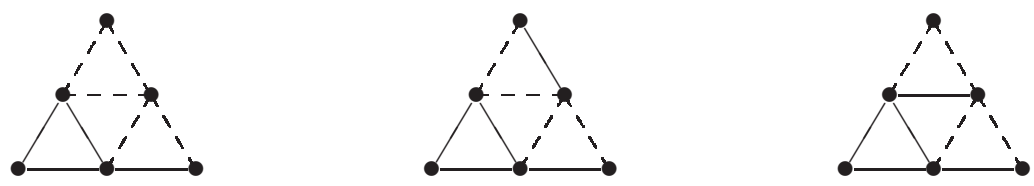

The first one gives the contribution $\frac{1}{x-1} T_{2, n} T_{1, n} T_{0, n}$; both the second and the third one give $\frac{1}{x-1} T_{2, n} T_{1, n}^{2}$. All these terms have to be multiplied by 2 because of the possible reflections. The three following configurations give the terms $\frac{1}{x-1} T_{1, n}^{3}, \frac{1}{x-1} T_{1, n}^{2} T_{0, n}$ and $\frac{1}{x-1} T_{2, n} T_{1, n}^{2}$, respectively.
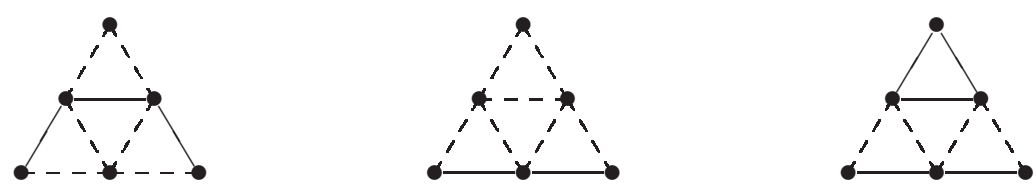

Finally, the two following configurations give $\frac{2}{x-1} T_{1, n}^{3}$ and $\frac{1}{x-1} T_{1, n}^{3}$, respectively (for the picture on the left, we have to take into account a possible reflection).
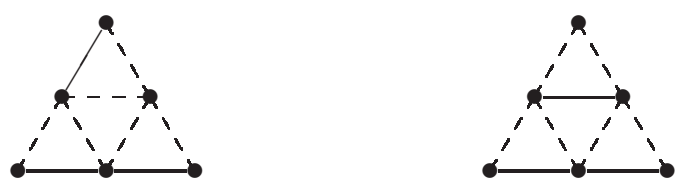
This completes the proof of Equation (4). Next, let us consider the contributions to the polynomial $T_{0, n+1}(x, y)$. The following pictures represent the only cases producing subgraphs of the first type.
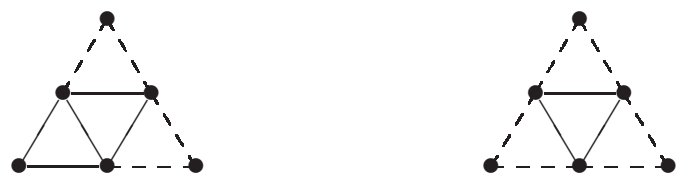

These configurations give the term $(y-1)\left(3 T_{2, n} T_{1, n}^{2}+T_{1, n}^{3}\right)$. Next, look at the following pictures.
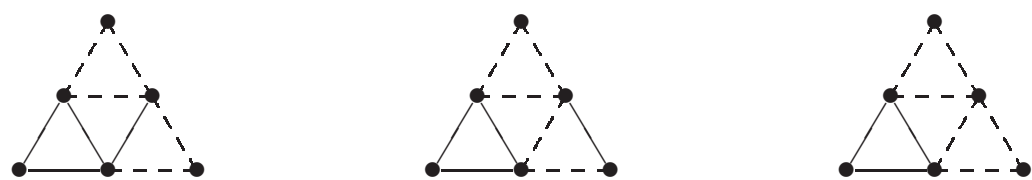

These configurations produce the terms $\frac{6}{x-1} T_{2, n} T_{1, n} T_{0, n}$ (reflections and rotations), then still $\frac{6}{x-1} T_{2, n} T_{1, n} T_{0, n}$ (reflections and rotations) and $\frac{3}{x-1} T_{2, n} T_{0, n}^{2}$ (only rotations), respectively. The three following configurations give the contribution $\frac{1}{x-1}\left(6 T_{1, n}^{3}+6 T_{1, n}^{3}+6 T_{1, n}^{2} T_{0, n}\right)$.
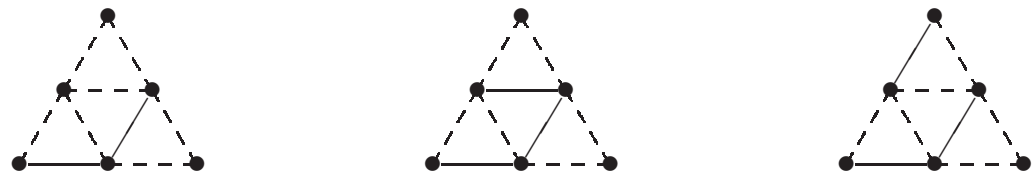

Next, look at the three following pictures. The first one contributes by $\frac{6}{x-1} T_{1, n}^{2} T_{0, n}$, the second one by $\frac{2}{x-1} T_{1, n}^{3}$ (we only have to take into account one reflection this time) and the third one by $\frac{6}{x-1} T_{1, n}^{2} T_{0, n}$.
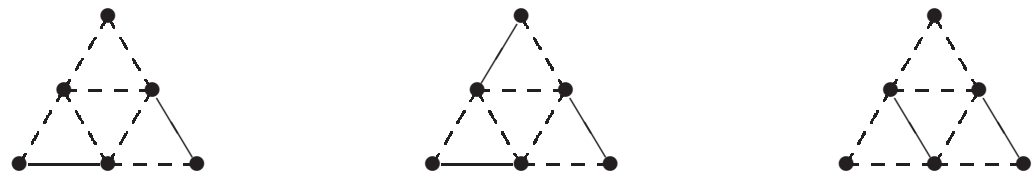

Each one of the following configurations produce a term $\frac{3}{x-1} T_{1, n}^{2} T_{0, n}$, since one has to consider rotations.
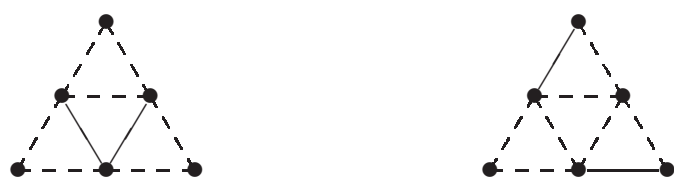

Finally, the three following pictures give $\frac{3}{x-1} T_{1, n} T_{0, n}^{2}, \frac{6}{x-1} T_{1, n} T_{0, n}^{2}$ and $\frac{1}{x-1} T_{0, n}^{3}$, respectively.
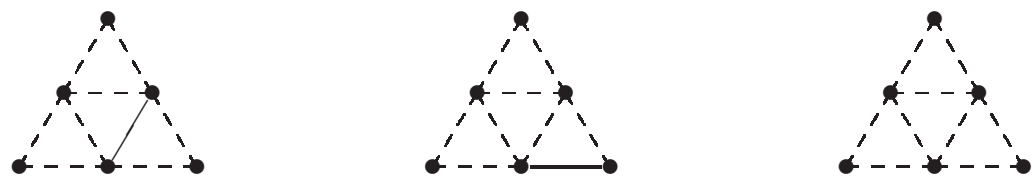
This proves Equation (5).

The following lemma can be easily proven by induction, using Equations (44) and (5D).

Lemma 3.2. For each $n \geq 1, x-1$ divides $T_{1, n}(x, y)$ and $(x-1)^{2}$ divides $T_{0, n}(x, y)$ in $\mathbb{Z}[x, y]$.

As a consequence, we can write

$$
T_{1, n}(x, y)=(x-1) N_{n}(x, y) \quad \text { and } \quad T_{0, n}(x, y)=(x-1)^{2} M_{n}(x, y),
$$

with $N_{n}(x, y)$ and $M_{n}(x, y) \in \mathbb{Z}[x, y]$.

Using Equation (6) for $T_{1, n}(x, y)$ and $T_{0, n}(x, y)$, Equations (3), (44), (5) can be rewritten as

$$
\begin{aligned}
& T_{2, n+1}(x, y)=(y-1) T_{2, n}^{3}+3(x-1) T_{2, n} N_{n}^{2}+6 T_{2, n}^{2} N_{n} \\
& N_{n+1}(x, y)=(y-1) T_{2, n}^{2} N_{n}+T_{2, n}^{2} M_{n}+7 T_{2, n} N_{n}^{2} \\
&+(x-1)\left(2 T_{2, n} N_{n} M_{n}+4 N_{n}^{3}\right)+(x-1)^{2} N_{n}^{2} M_{n} \\
& M_{n+1}(x, y)=(y-1)\left((x-1) N_{n}^{3}+3 T_{2, n} N_{n}^{2}\right)+12 T_{2, n} N_{n} M_{n}+14 N_{n}^{3} \\
&+(x-1)\left(3 T_{2, n} M_{n}^{2}+24 N_{n}^{2} M_{n}\right)+9(x-1)^{2} N_{n} M_{n}^{2}+(x-1)^{3} M_{n}^{3},
\end{aligned}
$$

with initial conditions

$$
T_{2,1}(x, y)=y+2 \quad N_{1}(x, y)=M_{1}(x, y)=1 .
$$

These reduced equations turn out to be very useful for many computations that can be done by evaluating the Tutte polynomial in special points of the line $x=1$. Let us start by writing the reliability polynomial $R\left(\Gamma_{n}, p\right)$.

Proposition 3.3. For each $n \geq 1$, the reliability polynomial $R\left(\Gamma_{n}, p\right)$ is given by

$$
R\left(\Gamma_{n}, p\right)=p^{\frac{3^{n}+1}{2}}(1-p)^{\frac{3^{n}-1}{2}} T_{n}\left(1, \frac{1}{1-p}\right),
$$

with $T_{n}\left(1, \frac{1}{1-p}\right)=T_{2, n}\left(1, \frac{1}{1-p}\right)$ and

$$
T_{2, n+1}\left(1, \frac{1}{1-p}\right)=\frac{p}{1-p} T_{2, n}^{3}+6 T_{2, n}^{2} N_{n}
$$

$$
N_{n+1}\left(1, \frac{1}{1-p}\right)=\frac{p}{1-p} T_{2, n}^{2} N_{n}+T_{2, n}^{2} M_{n}+7 T_{2, n} N_{n}^{2}
$$

$$
M_{n+1}\left(1, \frac{1}{1-p}\right)=\frac{3 p}{1-p} T_{2, n} N_{n}^{2}+12 T_{2, n} N_{n} M_{n}+14 N_{n}^{3}
$$

with initial conditions

$$
T_{2,1}\left(1, \frac{1}{1-p}\right)=\frac{3-2 p}{1-p} \quad N_{1}\left(1, \frac{1}{1-p}\right)=M_{1}\left(1, \frac{1}{1-p}\right)=1 .
$$

Proof. One has $T_{n}\left(1, \frac{1}{1-p}\right)=T_{2, n}\left(1, \frac{1}{1-p}\right)$, since $T_{1, n}(1, y)=T_{0, n}(1, y)=0$, for every $y \in \mathbb{R}$ (see Lemma 3.2). Then, it suffices to apply (1) of Theorem 2.6 and use Equations (7), (8) and (91). 
Remark 3.4. The analytic property $T_{1, n}(1, y)=T_{0, n}(1, y)=0$ has the following geometric interpretation. The only nontrivial terms in the sum (11) of Definition 2.2, for $x=1$, correspond to subgraphs $A$ such that $r\left(\Gamma_{n}\right)-r(A)=0$. Since $|V(A)|=\left|V\left(\Gamma_{n}\right)\right|$, this means $k(A)=k\left(\Gamma_{n}\right)=$ 1 and so $A$ must be a connected spanning subgraph of $\Gamma_{n}$; this implies $A \in D_{2, n}$.

Proposition 3.5. The complexity $\tau\left(\Gamma_{n}\right)$ is given by $T_{n}(1,1)=T_{2, n}(1,1)$, where

$$
\begin{gathered}
T_{2, n+1}(1,1)=6 T_{2, n}^{2} N_{n} \\
N_{n+1}(1,1)=T_{2, n}^{2} M_{n}+7 T_{2, n} N_{n}^{2} \\
M_{n+1}(1,1)=12 T_{2, n} N_{n} M_{n}+14 N_{n}^{3},
\end{gathered}
$$

with initial conditions

$$
T_{2,1}(1,1)=3 \quad N_{1}(1,1)=M_{1}(1,1)=1 .
$$

Proof. One can compute $T_{n}(1,1)$ by evaluating $T_{n}\left(1, \frac{1}{1-p}\right)=T_{2, n}\left(1, \frac{1}{1-p}\right)$ in $p=0$, using Equations (10), (11) and (12).

Remark 3.6. These equations coincide with the relations obtained in [11, 13, 26, without using Tutte polynomials. More precisely, one can find in [11, Theorem 3.1] and [13, Corollary 2.3]:

(1) $T_{n}(1,1)=\tau\left(\Gamma_{n}\right)=2^{\frac{3^{n-1}-1}{2}} 3^{\frac{3^{n}+2 n-1}{4}} 5^{\frac{3^{n-1}-2 n+1}{4}}$;

(2) $N_{n}(1,1)=2^{\frac{3^{n-1}-1}{2}} 3^{\frac{3^{n}-2 n-1}{4}} 5^{\frac{3^{n-1}+2 n-3}{4}}$;

(3) $M_{n}(1,1)=2^{\frac{3^{n-1}-1}{2}} 3^{\frac{3^{n}-6 n+3}{4}} 5^{\frac{3^{n-1}+6 n-7}{4}}$.

Then, the asymptotic growth constant of the spanning trees of $\Gamma_{n}$ is

$$
\lim _{n \rightarrow \infty} \frac{\log \left(\tau\left(\Gamma_{n}\right)\right)}{\left|V\left(\Gamma_{n}\right)\right|}=\frac{1}{3} \log 2+\frac{1}{2} \log 3+\frac{1}{6} \log 5 .
$$

Evaluating $T_{n}\left(1, \frac{1}{1-p}\right)$ in $p=\frac{1}{2}$ gives the number of connected spanning subgraphs of $\Gamma_{n}$.

Proposition 3.7. The number of connected spanning subgraphs of $\Gamma_{n}$ is given by $T_{n}(1,2)=$ $T_{2, n}(1,2)$, with

$$
\begin{gathered}
T_{2, n+1}(1,2)=T_{2, n}^{3}+6 T_{2, n}^{2} N_{n} \\
N_{n+1}(1,2)=T_{2, n}^{2} N_{n}+T_{2, n}^{2} M_{n}+7 T_{2, n} N_{n}^{2} \\
M_{n+1}(1,2)=3 T_{2, n} N_{n}^{2}+12 T_{2, n} N_{n} M_{n}+14 N_{n}^{3},
\end{gathered}
$$

with initial conditions

$$
T_{2,1}(1,2)=4 \quad N_{1}(1,2)=M_{1}(1,2)=1 .
$$

Proof. By Lemma 3.2, one has $T_{1, n}(1, y)=T_{0, n}(1, y)=0$, for every $y \in \mathbb{R}$. Therefore $T_{n}(1,2)=$ $T_{2, n}(1,2)$ and it suffices to apply Formula (2) of Theorem 2.5.

Remark 3.8. This specialization of $T_{n}(x, y)$ returns the equations obtained in [10] without using the Tutte polynomial.

Another interesting computation concerns the number of spanning forests of the Sierpiński graph $\Gamma_{n}$. 
Proposition 3.9. The number of spanning forests of $\Gamma_{n}$ is given by

$$
T_{n}(2,1)=T_{2, n}(2,1)+3 N_{n}(2,1)+M_{n}(2,1),
$$

where

$$
\begin{gathered}
T_{2, n+1}(2,1)=6 T_{2, n}^{2} N_{n}+3 T_{2, n} N_{n}^{2} \\
N_{n+1}(2,1)=T_{2, n}^{2} M_{n}+7 T_{2, n} N_{n}^{2}+2 T_{2, n} N_{n} M_{n}+4 N_{n}^{3}+N_{n}^{2} M_{n} \\
M_{n+1}(2,1)=12 T_{2, n} N_{n} M_{n}+3 T_{2, n} M_{n}^{2}+14 N_{n}^{3}+24 N_{n}^{2} M_{n}+9 N_{n} M_{n}^{2}+M_{n}^{3},
\end{gathered}
$$

with initial conditions

$$
T_{2,1}(2,1)=3 \quad N_{1}(2,1)=M_{1}(2,1)=1 .
$$

Proof. It suffices to apply Formula (3) of Theorem 2.5 and to observe that $T_{1, n}(2, y)=N_{n}(2, y)$ and $T_{0, n}(2, y)=M_{n}(2, y)$, for each $y \in \mathbb{R}$.

Remark 3.10. This specialization of $T_{n}(x, y)$ returns the equations obtained in [9] without using the Tutte polynomial.

Next, we explicitly verify that, by evaluating the Tutte polynomial of $\Gamma_{n}$ in $(2,2)$, one gets $2^{\left|E\left(\Gamma_{n}\right)\right|}$ (see Formula (4) of Theorem 2.5).

Proposition 3.11. For each $n \geq 1$, one has $T_{n}(2,2)=2^{\left|E\left(\Gamma_{n}\right)\right|}=2^{3^{n}}$.

Proof. We prove the assertion by induction. For $n=1$, we have $T_{1}(2,2)=8=2^{3}=2^{\left|E\left(\Gamma_{1}\right)\right|}$. Then, we recall that $\left|E\left(\Gamma_{n}\right)\right|=3^{n}$, therefore $\left|E\left(\Gamma_{n+1}\right)\right|=3^{n+1}=3\left|E\left(\Gamma_{n}\right)\right|$. An easy computation shows that $T_{n+1}(2,2)=T_{n}(2,2)^{3}$; therefore, $T_{n+1}(2,2)=T_{n}(2,2)^{3}=\left(2^{\left|E\left(\Gamma_{n}\right)\right|}\right)^{3}=2^{3\left|E\left(\Gamma_{n}\right)\right|}=$ $2^{\left|E\left(\Gamma_{n+1}\right)\right|}$.

Finally, by evaluating the Tutte polynomial of $\Gamma_{n}$ in $(2,0)$, we investigate the number of acyclic orientations of $\Gamma_{n}$.

Proposition 3.12. The number of acyclic orientations on $\Gamma_{n}$ is $T_{n}(2,0)$, with

$$
T_{n+1}(2,0)=T_{n}(2,0)^{3}-2\left(T_{2, n}(2,0)+N_{n}(2,0)\right)^{3}
$$

and

$$
\begin{gathered}
T_{2, n+1}(2,0)=-T_{2, n}^{3}+6 T_{2, n}^{2} N_{n}+3 T_{2, n} N_{n}^{2} \\
N_{n+1}(2,0)=-T_{2, n}^{2} N_{n}+T_{2, n}^{2} M_{n}+7 T_{2, n} N_{n}^{2}+2 T_{2, n} N_{n} M_{n}+4 N_{n}^{3}+N_{n}^{2} M_{n} \\
M_{n+1}(2,0)=-3 T_{2, n} N_{n}^{2}+12 T_{2, n} N_{n} M_{n}+3 T_{2, n} M_{n}^{2}+13 N_{n}^{3}+24 N_{n}^{2} M_{n}+9 N_{n} M_{n}^{2}+M_{n}^{3},
\end{gathered}
$$

with initial conditions

$$
T_{2,1}(2,0)=2 \quad N_{1}(2,0)=M_{1}(2,0)=1 .
$$

Proof. It suffices to apply Formula (5) of Theorem 2.5. Then, one can directly verify that $T_{2, n+1}(2,0)+3 T_{1, n+1}(2,0)+T_{0, n+1}(2,0)$ can be rewritten as $T_{n}(2,0)^{3}-2\left(T_{2, n}(2,0)+N_{n}(2,0)\right)^{3}$. 
Remark 3.13. In [8], the author obtains recursively the number $f(n)$ of acyclic orientations of $\Gamma_{n}$ as a sum of four contributions, namely $f(n)=6 a(n)+6 b(n)+6 c(n)+d(n)$. On the other hand, via the Tutte polynomial, we need to introduce only three contributions, corresponding to $T_{2, n}(2,0), N_{n}(2,0), M_{n}(2,0)$. Moreover, it is not difficult to show by induction that the following correspondences hold:

$T_{2, n}(2,0)=2 a(n)+b(n) \quad T_{1, n}(2,0)=a(n)+b(n)+c(n) \quad T_{0, n}(2,0)=a(n)+2 b(n)+3 c(n)+d(n)$.

Next, we study the chromatic polynomial of $\Gamma_{n}$.

Proposition 3.14. For each $n \geq 1$, the chromatic polynomial $\chi_{n}(\lambda)$ of the Sierpinsski graph $\Gamma_{n}$ is

$$
\chi_{n}(\lambda)=(-1)^{\frac{3^{n}+1}{2}} \lambda P_{n}(\lambda),
$$

where $P_{n}(\lambda)=P_{2, n}(\lambda)+3 P_{1, n}(\lambda)+P_{0, n}(\lambda)$, and

$$
\begin{gathered}
P_{2, n+1}(\lambda)=-P_{2, n}^{3}-\frac{1}{\lambda}\left(6 P_{2, n}^{2} P_{1, n}+3 P_{2, n} P_{1, n}^{2}\right) \\
P_{1, n+1}(\lambda)=-P_{2, n}^{2} P_{1, n}-\frac{1}{\lambda}\left(P_{2, n}^{2} P_{0, n}+7 P_{2, n} P_{1, n}^{2}\right. \\
\left.+\quad 2 P_{2, n} P_{1, n} P_{0, n}+4 P_{1, n}^{3}+P_{1, n}^{2} P_{0, n}\right) \\
P_{0, n+1}(\lambda)=-\left(3 P_{2, n} P_{1, n}^{2}+P_{1, n}^{3}\right)-\frac{1}{\lambda}\left(12 P_{2, n} P_{1, n} P_{0, n}\right. \\
\left.+\quad 3 P_{2, n} P_{0, n}^{2}+14 P_{1, n}^{3}+24 P_{1, n}^{2} P_{0, n}+9 P_{1, n} P_{0, n}^{2}+P_{0, n}^{3}\right),
\end{gathered}
$$

with initial conditions

$$
P_{2,1}(\lambda)=2 \quad P_{1,1}(\lambda)=-\lambda \quad P_{0,1}(\lambda)=\lambda^{2} .
$$

Proof. It is an easy consequence of Equation (2) of Theorem 2.6, with the convention $P_{i, n}(\lambda)=$ $T_{i, n}(1-\lambda, 0)$, for each $n \geq 1$ and $i=0,1,2$.

It is known that the chromatic number $\chi\left(\Gamma_{n}\right)$ is 3 . Using the Tutte polynomial, we are able to prove the following stronger result about the colorability of $\Gamma_{n}$.

Proposition 3.15. The graph $\Gamma_{n}$ is uniquely 3-colorable.

Proof. It is easy to show, by induction, that for each $n \geq 1$ one has:

$$
P_{2, n}(3)=(-1)^{n+1} 2 \quad P_{1, n}(3)=(-1)^{n} 3 \quad P_{0, n}(3)=(-1)^{n+1} 9 .
$$

Therefore, $P_{n}(3)=(-1)^{n+1} 2$ and so $\chi_{n}(3)=(-1)^{\frac{3^{n}+1}{2}+n+1} 6=6$, showing that $\Gamma_{n}$ is uniquely 3 -colorable (up to permutation of the colors).

Remark 3.16. The same result has been proven in [20, Theorem 3.1], where the author uses the stronger induction assumption that $\Gamma_{n}$ is uniquely 3-colorable and in every 3-coloring the outmost vertices have different colors.

We end this section by investigating the relationship between the evaluation of the Tutte polynomial of the Sierpiński graph $\Gamma_{n}$ on the hyperbola $(x-1)(y-1)=2$ and the partition function of the Ising model on the same graph. In [14, Theorem 3.5], the partition function of the Ising model on $\Gamma_{n}$ has been described as

$$
Z_{n}=2^{\frac{3^{n}+3}{2}} \cosh (\beta J)^{3^{n}} \Phi_{n}(\tanh (\beta J)),
$$


with

$$
\Phi_{n}(z)=z^{\frac{3^{n}}{2}} \prod_{k=1}^{n} \phi_{k}^{3^{n-k}}(z)\left(\phi_{n+1}(z)-1\right),
$$

where $\phi_{1}(z)=\frac{z+1}{z^{1 / 2}}, \phi_{2}(z)=\frac{z^{2}+1}{z}$ and $\phi_{k}(z)=\phi_{k-1}^{2}(z)-3 \phi_{k-1}(z)+4$, for each $k \geq 3$.

Theorem 3.17. For each $n \geq 1$, one has

$$
2\left(e^{2 \beta J}-1\right)^{\left|V\left(\Gamma_{n}\right)\right|-1} e^{-\beta J\left|E\left(\Gamma_{n}\right)\right|} T_{n}\left(\frac{e^{2 \beta J}+1}{e^{2 \beta J}-1}, e^{2 \beta J}\right)=Z_{n} .
$$

Proof. Recall that $\left|E\left(\Gamma_{n}\right)\right|=3^{n}$ and $\left|V\left(\Gamma_{n}\right)\right|=\frac{3^{n}+3}{2}$. Let $e^{\beta J}=t$, so that Equation (13) can be written as

$$
\frac{2\left(t^{2}-1\right)^{\frac{3^{n}+1}{2}}}{t^{3^{n}}} T_{n}\left(\frac{t^{2}+1}{t^{2}-1}, t^{2}\right)=2^{\frac{3^{n}+3}{2}}\left(\frac{t^{2}+1}{2 t}\right)^{3^{n}} \Phi_{n}\left(\frac{t^{2}-1}{t^{2}+1}\right)
$$

or, more explicitly,

$$
\frac{2\left(t^{2}-1\right)^{\frac{3^{n}+1}{2}}}{t^{3^{n}}} T_{n}\left(\frac{t^{2}+1}{t^{2}-1}, t^{2}\right)=\left.2^{\frac{3^{n}+3}{2}}\left(\frac{t^{2}+1}{2 t}\right)^{3^{n}}\left(\frac{t^{2}-1}{t^{2}+1}\right)^{\frac{3^{n}}{2}} \prod_{k=1}^{n} \phi_{k}^{3^{n-k}}(z)\left(\phi_{n+1}(z)-1\right)\right|_{z=\frac{t^{2}-1}{t^{2}+1}} .
$$

In order to prove this equation we put, for each $n \geq 1$ :

$$
A_{n}(x, y)=T_{2, n}(x, y)+T_{1, n}(x, y) \quad B_{n}(x, y)=2 T_{1, n}(x, y)+T_{0, n}(x, y)
$$

and

$$
C_{n}=\frac{2\left(t^{2}-1\right)^{\frac{3^{n}+1}{2}}}{t^{3^{n}}} \quad D_{n}=2^{\frac{3^{n}+3}{2}}\left(\frac{t^{2}+1}{2 t}\right)^{3^{n}}\left(\frac{t^{2}-1}{t^{2}+1}\right)^{\frac{3^{n}}{2}}
$$

Observe that

$$
T_{n}(x, y)=A_{n}(x, y)+B_{n}(x, y)
$$

and

$$
C_{n+1}=\frac{C_{n}^{3}}{4\left(t^{2}-1\right)} \quad D_{n+1}=\frac{D_{n}^{3}}{8} .
$$

Therefore, to prove the required equation is equivalent to prove the following equalities:

$$
\begin{gathered}
C_{n} A_{n}\left(\frac{t^{2}+1}{t^{2}-1}, t^{2}\right)=\left.D_{n} \prod_{k=1}^{n} \phi_{k}^{3^{n-k}}(z)\right|_{z=\frac{t^{2}-1}{t^{2}+1}} \\
C_{n} B_{n}\left(\frac{t^{2}+1}{t^{2}-1}, t^{2}\right)=\left.D_{n} \prod_{k=1}^{n} \phi_{k}^{3^{n-k}}(z)\left(\phi_{n+1}(z)-2\right)\right|_{z=\frac{t^{2}-1}{t^{2}+1}} .
\end{gathered}
$$

We can prove them by induction, observing that Equations (3), (44) and (5), evaluated on the hyperbola $(x-1)(y-1)=2$, give:

$$
\begin{gathered}
A_{n+1}=\frac{1}{2}(y-1) A_{n}^{2}\left(2 A_{n}+B_{n}\right) \\
B_{n+1}=\frac{1}{2}(y-1) B_{n}\left(2 A_{n}+B_{n}\right)\left(A_{n}+B_{n}\right),
\end{gathered}
$$

with initial conditions

$$
A_{1}\left(\frac{y+1}{y-1}, y\right)=\frac{y(y+1)}{y-1} \quad B_{1}\left(\frac{y+1}{y-1}, y\right)=\frac{4 y}{(y-1)^{2}} .
$$


Indeed, for $n=1$, one has

$$
C_{1} A_{1}\left(\frac{t^{2}+1}{t^{2}-1}, t^{2}\right)=\left.D_{1} \phi_{1}(z)\right|_{z=\frac{t^{2}-1}{t^{2}+1}}=\frac{2\left(t^{4}-1\right)}{t}
$$

and

$$
C_{1} B_{1}\left(\frac{t^{2}+1}{t^{2}-1}, t^{2}\right)=\left.D_{1} \phi_{1}(z)\left(\phi_{2}(z)-2\right)\right|_{z=\frac{t^{2}-1}{t^{2}+1}}=\frac{8}{t}
$$

thus, the assertion is true. Now

$$
\begin{aligned}
C_{n+1} A_{n+1} & =\frac{1}{8} C_{n}^{2} A_{n}^{2}\left(2 C_{n} A_{n}+C_{n} B_{n}\right) \\
& =\frac{1}{8} D_{n}^{3}\left(\prod_{k=1}^{n} \phi_{k}^{3^{n-k}}(z)\right)^{3}\left(2+\left(\phi_{n+1}(z)-2\right)\right) \\
& =D_{n+1} \prod_{k=1}^{n+1} \phi_{k}^{3^{n+1-k}}(z)
\end{aligned}
$$

Similarly, one has

$$
\begin{aligned}
C_{n+1} B_{n+1} & =\frac{1}{8} C_{n} B_{n}\left(2 C_{n} A_{n}+C_{n} B_{n}\right)\left(C_{n} A_{n}+C_{n} B_{n}\right) \\
& =\frac{1}{8} D_{n}^{3}\left(\prod_{k=1}^{n} \phi_{k}^{3^{n-k}}(z)\right)^{3}\left(\phi_{n+1}(z)-2\right)\left(2+\phi_{n+1}(z)-2\right)\left(1+\phi_{n+1}(z)-2\right) \\
& =D_{n+1} \prod_{k=1}^{n+1} \phi_{k}^{3^{n+1-k}}(z)\left(\phi_{n+1}^{2}(z)-3 \phi_{n+1}(z)+2\right) \\
& =D_{n+1} \prod_{k=1}^{n+1} \phi_{k}^{3^{n+1-k}}(z)\left(\phi_{n+2}(z)-2\right) .
\end{aligned}
$$

\section{The Tutte polynomial of the Schreier graphs of the Hanoi Towers group}

In this section, we study the Tutte polynomial of the Schreier graphs $\left\{\Sigma_{n}\right\}_{n \geq 1}$ of the Hanoi Towers group $H^{(3)}$. The strategy is still to use the self-similarity of the graphs in order to approach recursively the problem.

4.1. The Schreier graphs of the Hanoi Towers group. The Hanoi Towers groups $H^{(3)}$ is generated by the automorphisms of the ternary rooted tree having the following self-similar form:

$$
a=(01)(i d, i d, a) \quad b=(02)(i d, b, i d) \quad c=(12)(c, i d, i d),
$$

where (01),(02) and (12) are elements of the symmetric group $\operatorname{Sym}(3)$ acting on the set $X=$ $\{0,1,2\}$. Observe that $a, b, c$ are involutions. The associated Schreier graphs are self-similar in the sense of [27], that is, $\Sigma_{n}$ contains three copies of $\Sigma_{n-1}$ glued together by three edges, that we call special edges. Their endpoints will be called special vertices of $\Sigma_{n}$. These graphs can be recursively constructed via the following substitutional rules [18], 
Rule I
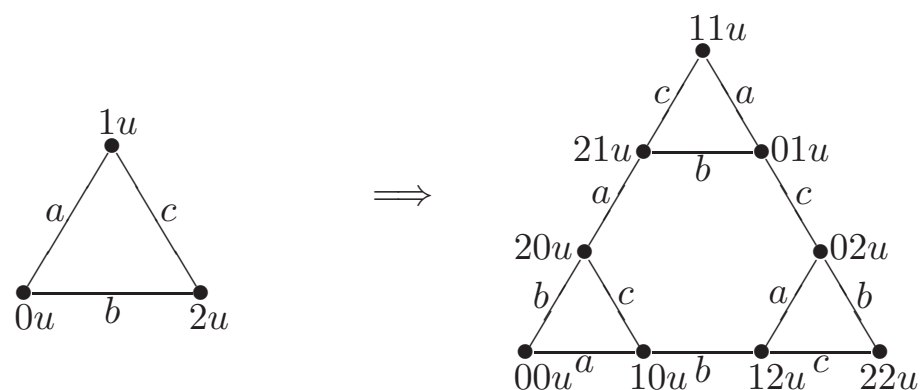

Rule II
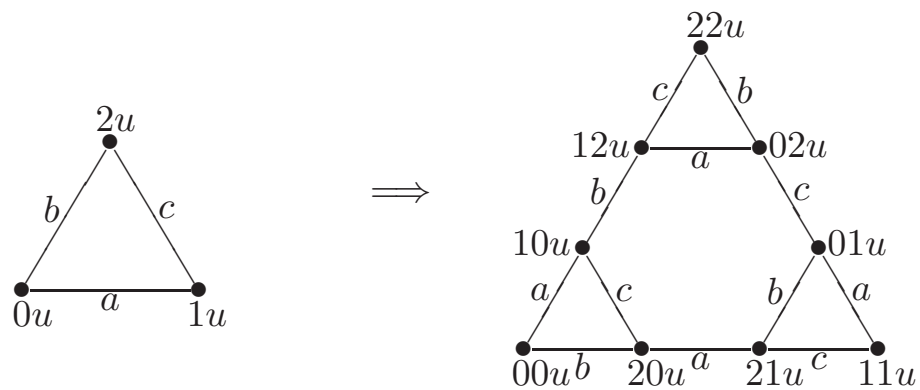

Rule III $\left.\left.\left.\left.\left.c\right|_{0 v} ^{0 u} \Longrightarrow c\right|_{00 v} ^{00 u} \operatorname{Rule~IV~}_{b} \Longrightarrow b\right|_{i v} ^{11 u} \operatorname{Rule~}\right|_{a} ^{2 u} \Longrightarrow a\right|_{2 v} ^{22 u}$

where the word $u$ in Rule I and Rule II can also be the empty word and the words $u$ and $v$ in Rules III, IV, V can also satisfy $u=v$ (in this case we get the three loops of $\Sigma_{n}$ ). The starting point is the Schreier graph $\Sigma_{1}$ of the first level. We also draw a picture of $\Sigma_{2}$.
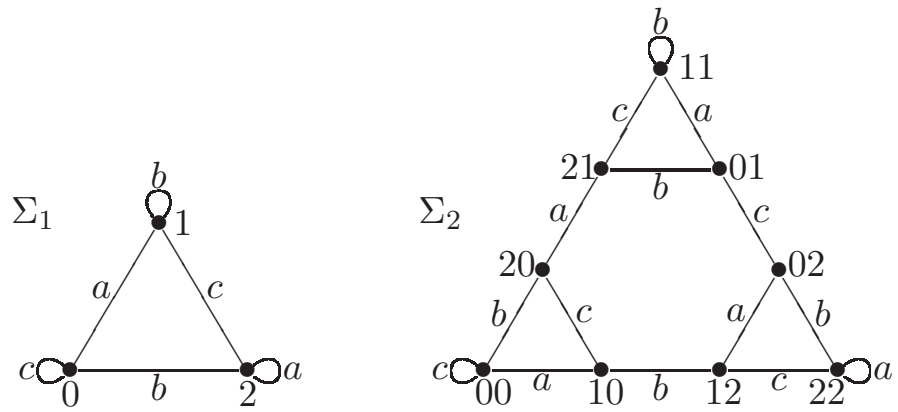

Remark 4.1. Observe that, for each $n \geq 1$, the graph $\Sigma_{n}$ has three loops, centered at the outmost vertices $0^{n}, 1^{n}$ and $2^{n}$, labelled by $c, b$ and $a$, respectively. This is an easy consequence of the definition of the generators $a, b$ and $c$ of $H^{(3)}$. Moreover, these are the only loops in $\Sigma_{n}$.

Remark 4.2. As we already mentioned in Section 3 , the graphs $\left\{\Sigma_{n}\right\}_{n \geq 1}$ are very close to the Sierpiński graphs $\left\{\Gamma_{n}\right\}_{n>1}$. Indeed, each $\Gamma_{n}$ can be obtained from $\Sigma_{n}$ by removing loops and contracting all the special edges of $\Sigma_{n}$ at each step.

4.2. The Tutte polynomial of $\Sigma_{n}$. In this section we study the Tutte polynomial of the graph $\Sigma_{n}$, considered without its loops. However, the presence of the three loops would change the polynomial only by the factor $y^{3}$ (see Definition 2.3). Moreover, for our purposes, we can forget the generator labelling of the edges of $\Sigma_{n}$, as well as the word labelling of its vertices, and we can regard it as an unlabelled graph. We represent the self-similar structure of $\Sigma_{n}$ by the following picture. 


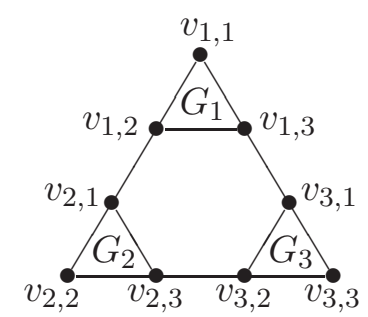

More precisely, the graph $\Sigma_{n}$ is the union of three copies $G_{1}, G_{2}$ and $G_{3}$ of $\Sigma_{n-1}$, joint by the special edges. For each $i=1,2,3$, we denote by $v_{i, j}$, with $j=1,2,3$, the upmost, the leftmost and the rightmost vertex of $G_{i}$, respectively. Moreover, it is not difficult to prove by induction the following equalities:

$$
\left|V\left(\Sigma_{n}\right)\right|=3^{n} \quad\left|E\left(\Sigma_{n}\right)\right|=\frac{3^{n+1}-3}{2} .
$$

Note that for $n=1, \Sigma_{1}=\Gamma_{1}$, and so everything is already known. As for the Sierpiński graphs, we introduce the following partition of the set of the spanning subgraphs of $\Sigma_{n}$ :

- $F_{2, n}$ denotes the set of spanning subgraphs of $\Sigma_{n}$, where the three outmost vertices belong to the same connected component;

- $F_{1, n}^{u}$ denotes the set of spanning subgraphs of $\Sigma_{n}$, where the leftmost and rightmost vertices belong to the same connected component, and the upmost one belongs to a different connected component. Similarly, by rotation, $F_{1, n}^{r}$ (respectively $F_{1, n}^{l}$ ) denotes the set of spanning subgraphs of $\Sigma_{n}$, where the rightmost (respectively leftmost) vertex is not in the same connected component containing the two other outmost vertices;

- $F_{0, n}$ denotes the set of spanning subgraphs of $\Sigma_{n}$, where the three outmost vertices belong to three different connected components.

As in Section 3, to draw a subgraph of $\Sigma_{n}$ of the previous types, we will use the following notation.
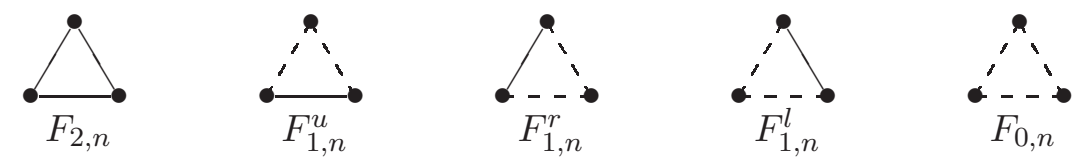

Observe that

$$
F_{2, n} \sqcup F_{1, n}^{u} \sqcup F_{1, n}^{r} \sqcup F_{1, n}^{l} \sqcup F_{0, n}
$$

is a partition of the set of spanning subgraphs of $\Sigma_{n}$, for each $n \geq 1$. Next, let us simply denote by $H_{n}(x, y)$ the Tutte polynomial $T\left(\Sigma_{n} ; x, y\right)$ of $\Sigma_{n}$ and define, for every $n \geq 1$, the following polynomials:

$$
\begin{aligned}
& \text { - } H_{2, n}(x, y)=\sum_{A \in F_{2, n}}(x-1)^{r\left(\Sigma_{n}\right)-r(A)}(y-1)^{n(A)} ; \\
& \text { - } H_{1, n}^{u}(x, y)=\sum_{A \in F_{1, n}^{u}}(x-1)^{r\left(\Sigma_{n}\right)-r(A)}(y-1)^{n(A)} ; \\
& \text { - } H_{0, n}(x, y)=\sum_{A \in F_{0, n}}(x-1)^{r\left(\Sigma_{n}\right)-r(A)}(y-1)^{n(A)}
\end{aligned}
$$

Similarly, we define $H_{1, n}^{r}(x, y)$ and $H_{1, n}^{l}(x, y)$, by taking sums over $F_{1, n}^{r}$ and $F_{1, n}^{l}$, respectively. Note that, by the rotational-invariance of the graph $\Sigma_{n}$, one has

$$
H_{1, n}^{u}(x, y)=H_{1, n}^{r}(x, y)=H_{1, n}^{l}(x, y),
$$


so that we can simply use the notation $H_{1, n}(x, y)$ to denote one of these three polynomials. According with Definition 2.2 of the Tutte polynomial, we have:

$$
H_{n}(x, y)=H_{2, n}(x, y)+3 H_{1, n}(x, y)+H_{0, n}(x, y) .
$$

Also in this case, we give a recursive formula for $H_{n}(x, y)$, providing recursive formulas for $H_{2, n}(x, y), H_{1, n}(x, y)$ and $H_{0, n}(x, y)$ (Theorem 4.3). The main difference with respect to the case of the Sierpiński graphs is that, now, a spanning subgraph $A$ of $\Sigma_{n+1}$ is not determined by its restrictions $A_{1}, A_{2}$ and $A_{3}$ to the three copies $G_{1}, G_{2}$ and $G_{3}$ of $\Sigma_{n}$. In fact, the three special edges do not belong to any of the copies of the $\Sigma_{n}$. Therefore, in this case, we need to specify how many special edges belong to the subgraph $A$ of $\Sigma_{n+1}$. Once we fix them, then we have the same correspondence as before, i.e., a spanning subgraph $A$ in $\Sigma_{n+1}$ is determined by the special edges that it contains and by its restrictions to the three copies $G_{1}, G_{2}$ and $G_{3}$ of $\Sigma_{n}$.

Therefore, Equation (1) of Definition 2.2 can be rewritten as

$$
H_{n+1}(x, y)=\sum_{A_{i} \subseteq G_{i}, i=1,2,3}(x-1)^{r\left(\Sigma_{n+1}\right)-r(A)}(y-1)^{n(A)} .
$$

Firstly, observe that $r\left(\Sigma_{n+1}\right)=3 r\left(\Sigma_{n}\right)+2$ and $|V(A)|=\left|V\left(A_{1}\right)\right|+\left|V\left(A_{2}\right)\right|+\left|V\left(A_{3}\right)\right|$, for every spanning subgraph $A$ of $\Sigma_{n+1}$. Next, we have to understand how $r(A)$ and $n(A)$ depend on $r\left(A_{i}\right)$ and $n\left(A_{i}\right)$, for $i=1,2,3$. Note that the number of special edges belonging to $A$ plays a crucial role. Moreover, we still have to separately consider the case in which the special vertices belongs to the same connected components: this can only happen when all the special edges belong to $A$.

Case I: All the special edges are in A.

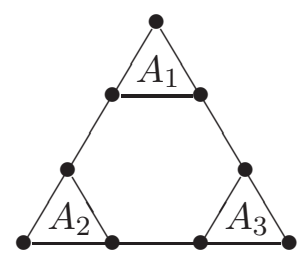

This case is analogous to the case of the Sierpiński graphs. If in the spanning subgraph $A$, obtained by the union of the special edges and $A_{1}, A_{2}$ and $A_{3}$, the special vertices are in the same connected component and, for each $i=1,2,3$, the vertices $v_{i, j}$ and $v_{i, k}$ are in the same connected component of $A_{i}$, for $j, k \neq i$, then it is easy to check that

$$
k(A)=k\left(A_{1}\right)+k\left(A_{2}\right)+k\left(A_{3}\right)-2 \quad \text { and } \quad r(A)=r\left(A_{1}\right)+r\left(A_{2}\right)+r\left(A_{3}\right)+2 .
$$

Moreover, one has

$$
\begin{aligned}
n(A) & =\left(\left|E\left(A_{1}\right)\right|+\left|E\left(A_{2}\right)\right|+\left|E\left(A_{3}\right)\right|+3\right)-\left(\left|V\left(A_{1}\right)\right|+\left|V\left(A_{2}\right)\right|+\left|V\left(A_{3}\right)\right|\right) \\
& +\left(k\left(A_{1}\right)+k\left(A_{2}\right)+k\left(A_{3}\right)-2\right) \\
& =n\left(A_{1}\right)+n\left(A_{2}\right)+n\left(A_{3}\right)+1 .
\end{aligned}
$$

Hence, for such a spanning subgraph $A$ of $\Sigma_{n+1}$ (of "first type"), one gets:

$$
r\left(\Sigma_{n+1}\right)-r(A)=\sum_{i=1}^{3}\left(r\left(\Sigma_{n}\right)-r\left(A_{i}\right)\right) \quad \text { and } \quad n(A)=n\left(A_{1}\right)+n\left(A_{2}\right)+n\left(A_{3}\right)+1
$$

and so

$$
(x-1)^{r\left(\Sigma_{n+1}\right)-r(A)}(y-1)^{n(A)}=(y-1) \prod_{i=1}^{3}(x-1)^{r\left(\Sigma_{n}\right)-r\left(A_{i}\right)}(y-1)^{n\left(A_{i}\right)} .
$$


If in the spanning subgraph $A$, obtained by the union of the special edges with $A_{1}, A_{2}$ and $A_{3}$, the vertices $v_{i, j}$ and $v_{i, k}$ in $A_{i}$ do not belong to the same connected component of $A_{i}$, for some $i=1,2,3$ and $j, k \neq i$, then it is easy to verify that

$$
k(A)=k\left(A_{1}\right)+k\left(A_{2}\right)+k\left(A_{3}\right)-3 \quad \text { and } \quad r(A)=r\left(A_{1}\right)+r\left(A_{2}\right)+r\left(A_{3}\right)+3 .
$$

Therefore, one has in this case

$$
\begin{aligned}
n(A) & =\left(\left|E\left(A_{1}\right)\right|+\left|E\left(A_{2}\right)\right|+\left|E\left(A_{3}\right)\right|+3\right)-\left(\left|V\left(A_{1}\right)\right|+\left|V\left(A_{2}\right)\right|+\left|V\left(A_{3}\right)\right|\right) \\
& +\left(k\left(A_{1}\right)+k\left(A_{2}\right)+k\left(A_{3}\right)-3\right) \\
& =n\left(A_{1}\right)+n\left(A_{2}\right)+n\left(A_{3}\right) .
\end{aligned}
$$

Hence, for such a spanning subgraph $A$ of $\Sigma_{n+1}$ (of "second type"), one gets:

$$
r\left(\Sigma_{n+1}\right)-r(A)=\sum_{i=1}^{3}\left(r\left(\Sigma_{n}\right)-r\left(A_{i}\right)\right)-1 \quad \text { and } \quad n(A)=n\left(A_{1}\right)+n\left(A_{2}\right)+n\left(A_{3}\right)
$$

and so

$$
(x-1)^{r\left(\Sigma_{n+1}\right)-r(A)}(y-1)^{n(A)}=\frac{1}{(x-1)} \prod_{i=1}^{3}(x-1)^{r\left(\Sigma_{n}\right)-r\left(A_{i}\right)}(y-1)^{n\left(A_{i}\right)} .
$$

Case II: Only two special edges are in A.

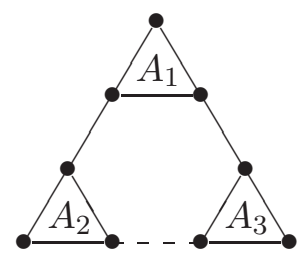

In this case

$$
k(A)=k\left(A_{1}\right)+k\left(A_{2}\right)+k\left(A_{3}\right)-2 \quad \text { and } \quad r(A)=r\left(A_{1}\right)+r\left(A_{2}\right)+r\left(A_{3}\right)+2 .
$$

One has in this case

$$
\begin{aligned}
n(A) & =\left(\left|E\left(A_{1}\right)\right|+\left|E\left(A_{2}\right)\right|+\left|E\left(A_{3}\right)\right|+2\right)-\left(\left|V\left(A_{1}\right)\right|+\left|V\left(A_{2}\right)\right|+\left|V\left(A_{3}\right)\right|\right) \\
& +\left(k\left(A_{1}\right)+k\left(A_{2}\right)+k\left(A_{3}\right)-2\right) \\
& =n\left(A_{1}\right)+n\left(A_{2}\right)+n\left(A_{3}\right) .
\end{aligned}
$$

Hence, for such a spanning subgraph $A$ of $\Sigma_{n+1}$ (of "third type"), one gets:

$$
r\left(\Sigma_{n+1}\right)-r(A)=\sum_{i=1}^{3}\left(r\left(\Sigma_{n}\right)-r\left(A_{i}\right)\right) \quad \text { and } \quad n(A)=n\left(A_{1}\right)+n\left(A_{2}\right)+n\left(A_{3}\right)
$$

and so

$$
(x-1)^{r\left(\Sigma_{n+1}\right)-r(A)}(y-1)^{n(A)}=\prod_{i=1}^{3}(x-1)^{r\left(\Sigma_{n}\right)-r\left(A_{i}\right)}(y-1)^{n\left(A_{i}\right)} .
$$


Case III: Only one special edge is in A.

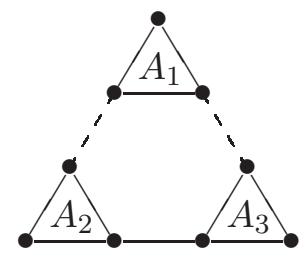

In this case

$$
k(A)=k\left(A_{1}\right)+k\left(A_{2}\right)+k\left(A_{3}\right)-1 \quad \text { and } \quad r(A)=r\left(A_{1}\right)+r\left(A_{2}\right)+r\left(A_{3}\right)+1 .
$$

Moreover, one has in this case

$$
\begin{aligned}
n(A) & =\left(\left|E\left(A_{1}\right)\right|+\left|E\left(A_{2}\right)\right|+\left|E\left(A_{3}\right)\right|+1\right)-\left(\left|V\left(A_{1}\right)\right|+\left|V\left(A_{2}\right)\right|+\left|V\left(A_{3}\right)\right|\right) \\
& +\left(k\left(A_{1}\right)+k\left(A_{2}\right)+k\left(A_{3}\right)-1\right) \\
& =n\left(A_{1}\right)+n\left(A_{2}\right)+n\left(A_{3}\right) .
\end{aligned}
$$

Hence, for such a spanning subgraph $A$ of $\Sigma_{n+1}$ (of "fourth type"), one gets:

$$
r\left(\Sigma_{n+1}\right)-r(A)=\sum_{i=1}^{3}\left(r\left(\Sigma_{n}\right)-r\left(A_{i}\right)\right)+1 \quad \text { and } \quad n(A)=n\left(A_{1}\right)+n\left(A_{2}\right)+n\left(A_{3}\right)
$$

and so

$$
(x-1)^{r\left(\Sigma_{n+1}\right)-r(A)}(y-1)^{n(A)}=(x-1) \prod_{i=1}^{3}(x-1)^{r\left(\Sigma_{n}\right)-r\left(A_{i}\right)}(y-1)^{n\left(A_{i}\right)} .
$$

Case IV: No special edge is in A.

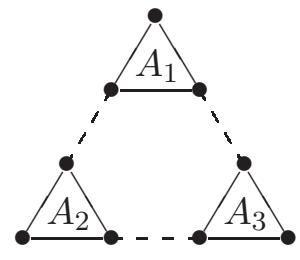

In this case

$$
k(A)=k\left(A_{1}\right)+k\left(A_{2}\right)+k\left(A_{3}\right) \quad \text { and } \quad r(A)=r\left(A_{1}\right)+r\left(A_{2}\right)+r\left(A_{3}\right) .
$$

Moreover, one has in this case

$$
\begin{aligned}
n(A) & =\left(\left|E\left(A_{1}\right)\right|+\left|E\left(A_{2}\right)\right|+\left|E\left(A_{3}\right)\right|\right)-\left(\left|V\left(A_{1}\right)\right|+\left|V\left(A_{2}\right)\right|+\left|V\left(A_{3}\right)\right|\right) \\
& +\left(k\left(A_{1}\right)+k\left(A_{2}\right)+k\left(A_{3}\right)\right) \\
& =n\left(A_{1}\right)+n\left(A_{2}\right)+n\left(A_{3}\right) .
\end{aligned}
$$

Hence, for such a spanning subgraph $A$ of $\Sigma_{n+1}$ (of "fifth type"), one gets:

$$
r\left(\Sigma_{n+1}\right)-r(A)=\sum_{i=1}^{3}\left(r\left(\Sigma_{n}\right)-r\left(A_{i}\right)\right)+2 \quad \text { and } \quad n(A)=n\left(A_{1}\right)+n\left(A_{2}\right)+n\left(A_{3}\right)
$$

and so

$$
(x-1)^{r\left(\Sigma_{n+1}\right)-r(A)}(y-1)^{n(A)}=(x-1)^{2} \prod_{i=1}^{3}(x-1)^{r\left(\Sigma_{n}\right)-r\left(A_{i}\right)}(y-1)^{n\left(A_{i}\right)} .
$$


Theorem 4.3. For each $n \geq 1$, the Tutte polynomial $H_{n}(x, y)$ of $\Sigma_{n}$ is given by

$$
H_{n}(x, y)=H_{2, n}(x, y)+3 H_{1, n}(x, y)+H_{0, n}(x, y)
$$

where the polynomials $H_{2, n}(x, y), H_{1, n}(x, y), H_{0, n}(x, y) \in \mathbb{Z}[x, y]$ satisfy the following recursive relations:

$$
\begin{aligned}
H_{2, n+1}(x, y) & =(y-1) H_{2, n}^{3}+\frac{1}{x-1}\left(6 H_{2, n}^{2} H_{1, n}+3 H_{2, n} H_{1, n}^{2}\right) \\
& +3 H_{2, n}^{3}+6 H_{2, n}^{2} H_{1, n}+3 H_{2, n} H_{1, n}^{2} .
\end{aligned}
$$

$$
\begin{aligned}
(15) H_{1, n+1}(x, y) & =(y-1) H_{2, n}^{2} H_{1, n} \\
& +\frac{1}{x-1}\left(H_{2, n}^{2} H_{0, n}+7 H_{2, n} H_{1, n}^{2}+2 H_{2, n} H_{1, n} H_{0, n}+4 H_{1, n}^{3}+H_{1, n}^{2} H_{0, n}\right) \\
& +7 H_{2, n}^{2} H_{1, n}+2 H_{2, n}^{2} H_{0, n}+14 H_{2, n} H_{1, n}^{2}+4 H_{2, n} H_{1, n} H_{0, n} \\
& +7 H_{1, n}^{3}+2 H_{1, n}^{2} H_{0, n}+(x-1)\left(H_{2, n}^{3}+5 H_{2, n}^{2} H_{1, n}+H_{2, n}^{2} H_{0, n}\right. \\
+ & \left.7 H_{2, n} H_{1, n}^{2}+2 H_{2, n} H_{1, n} H_{0, n}+3 H_{1, n}^{3}+H_{1, n}^{2} H_{0, n}\right) \\
& (y-1)\left(3 H_{2, n} H_{1, n}^{2}+H_{1, n}^{3}\right) \\
& +\frac{1}{(16) H_{0, n+1}(x, y)}\left(12 H_{2, n} H_{1, n} H_{0, n}+3 H_{2, n} H_{0, n}^{2}+14 H_{1, n}^{3}+24 H_{1, n}^{2} H_{0, n}\right. \\
+ & \left.9 H_{1, n} H_{0, n}^{2}+H_{0, n}^{3}\right)+3 H_{2, n}^{2} H_{0, n}+36 H_{2, n} H_{1, n}^{2}+42 H_{2, n} H_{1, n} H_{0, n} \\
+ & 9 H_{2, n} H_{0, n}^{2}+60 H_{1, n}^{3}+75 H_{1, n}^{2} H_{0, n}+27 H_{1, n} H_{0, n}^{2}+3 H_{0, n}^{3} \\
+ & (x-1)\left(12 H_{2, n}^{2} H_{1, n}+6 H_{2, n}^{2} H_{0, n}+60 H_{2, n} H_{1, n}^{2}+48 H_{2, n} H_{1, n} H_{0, n}\right. \\
+ & \left.9 H_{2, n} H_{0, n}^{2}+72 H_{1, n}^{3}+78 H_{1, n}^{2} H_{0, n}+27 H_{1, n} H_{0, n}^{2}+3 H_{0, n}^{3}\right) \\
+ & (x-1)^{2}\left(H_{2, n}^{3}+9 H_{2, n}^{2} H_{1, n}+3 H_{2, n}^{2} H_{0, n}+27 H_{2, n} H_{1, n}^{2}+18 H_{2, n} H_{1, n} H_{0, n}\right. \\
+ & \left.3 H_{2, n} H_{0, n}^{2}+27 H_{1, n}^{3}+27 H_{1, n}^{2} H_{0, n}+9 H_{1, n} H_{0, n}^{2}+H_{0, n}^{3}\right) \\
&
\end{aligned}
$$

with initial conditions

$$
H_{2,1}(x, y)=y+2 \quad H_{1,1}(x, y)=x-1 \quad H_{0,1}(x, y)=(x-1)^{2} .
$$

Proof. The proof follows the same strategy as in Theorem 3.1. Observe that in this case we have different powers of $(x-1)$ occurring, due to the different possible number of special edges belonging to a spanning subgraph.

For each $n \geq 1$, let us call $I_{n}$ the graph obtained by $\Sigma_{n}$ by contracting only the special edges joining the three copies of $\Sigma_{n-1}$, so that $I_{n}$ has the following structure.

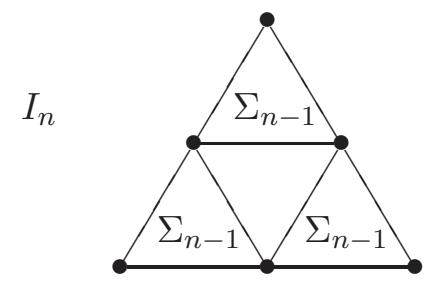


In other words, the graph $I_{n}$ can be regarded as a Sierpiński graph $\Gamma_{n}$, where each subgraph $G_{1}, G_{2}, G_{3}$ is isomorphic to $\Sigma_{n-1}$ and not to the graph $\Gamma_{n-1}$.

The following proposition establishes a relationship between the Tutte polynomial of $\Sigma_{n}$ and the Tutte polynomial of the Sierpinski graph $\Gamma_{n}$, via the introduction of the Tutte polynomial of $I_{n}$. More precisely, the following result holds.

Proposition 4.4. For each $n \geq 1$, one has

$$
H_{n+1}(x, y)=\left(x^{2}+x+1\right) H_{n}^{3}(x, y)+T\left(I_{n+1} ; x, y\right) .
$$

Proof. We prove the assertion by using Property (21) and the deletion-contraction property of the Tutte polynomial (see Definition 2.31). Let us start by choosing the bottom special edge in $\Sigma_{n+1}$ : then, by deletion and contraction, we have
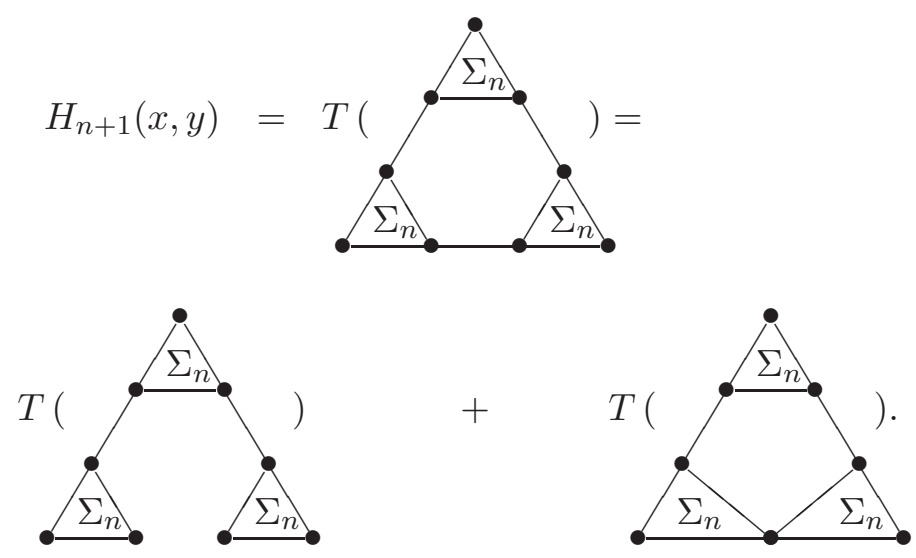

Next, in order to compute the Tutte polynomial, we can use Property (2) for the graph on the left and, for the graph on the right, we can apply again the deletion-contraction argument, with respect to the left special edge. Thus, we get:
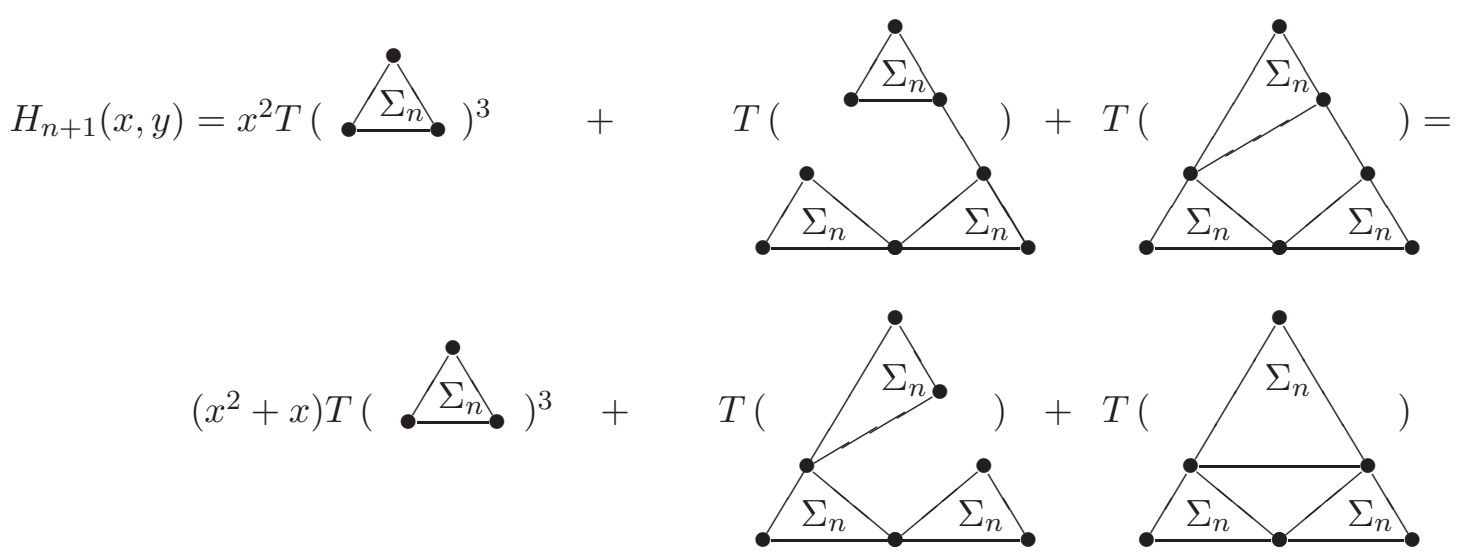

where the last equality is obtained by using Property (2) and then by applying the deletioncontraction argument with respect to the right special edge. Finally, we can apply again Property (2) for the middle graph, and then we can observe that the graph $I_{n+1}$ appeared on the right, so that we get: 


$$
H_{n+1}(x, y)=\left(x^{2}+x+1\right) T\left(\bullet \Sigma_{n}\right)^{3}+T(
$$

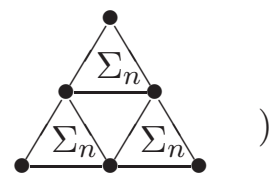

and so $H_{n+1}(x, y)=\left(x^{2}+x+1\right) H_{n}^{3}(x, y)+T\left(I_{n+1} ; x, y\right)$, as required.

Remark 4.5. Since the graph $I_{n+1}$ can be regarded as the Sierpiński graph $\Gamma_{n+1}$, where each subgraph $G_{1}, G_{2}, G_{3}$ is isomorphic to $\Sigma_{n}$, it is clear that $T\left(I_{n+1} ; x, y\right)$ is given by $T_{n+1}(x, y)$, obtained from Equations (3), (4) and (5), where $T_{2, n}, T_{1, n}$ and $T_{0, n}$ have to be replaced by $H_{2, n}$, $H_{1, n}$ and $H_{0, n}$, respectively. Moreover, the terms of $T\left(I_{n+1} ; x, y\right)$ in $H_{n+1}(x, y)$ are exactly the terms of $H_{n+1}(x, y)$ having a factor $(y-1)$ or $\frac{1}{x-1}$, i.e., the terms of first and second type corresponding to subgraphs of $\Sigma_{n+1}$ containing the three special edges.

The following lemma can be easily proven by induction, using Equations (15) and (16).

Lemma 4.6. For each $n \geq 1, x-1$ divides $H_{1, n}(x, y)$ and $(x-1)^{2}$ divides $H_{0, n}(x, y)$ in $\mathbb{Z}[x, y]$.

As a consequence, we can write

$$
H_{1, n}(x, y)=(x-1) N_{n}(x, y) \quad \text { and } \quad H_{0, n}(x, y)=(x-1)^{2} M_{n}(x, y),
$$

with $N_{n}(x, y)$ and $M_{n}(x, y) \in \mathbb{Z}[x, y]$.

Using (17) for $H_{1, n}(x, y)$ and $H_{0, n}(x, y)$, Equations (14), (15) and (16) can be rewritten as

$$
\begin{aligned}
N_{n+1}(x, y) & =(y-1) H_{2, n}^{2} N_{n}+H_{2, n}^{3}+7 H_{2, n}^{2} N_{n}+H_{2, n}^{2} M_{n}+7 H_{2, n} N_{n}^{2} \\
& +(x-1)\left(5 H_{2, n}^{2} N_{n}+2 H_{2, n}^{2} M_{n}+14 H_{2, n} N_{n}^{2}+2 H_{2, n} N_{n} M_{n}+4 N_{n}^{3}\right) \\
& +(x-1)^{2}\left(H_{2, n}^{2} M_{n}+7 H_{2, n} N_{n}^{2}+4 H_{2, n} N_{n} M_{n}+7 N_{n}^{3}+N_{n}^{2} M_{n}\right) \\
& +(x-1)^{3}\left(2 H_{2, n} N_{n} M_{n}+3 N_{n}^{3}+2 N_{n}^{2} M_{n}\right) \\
+ & (x-1)^{4} N_{n}^{2} M_{n}
\end{aligned}
$$

(20) $M_{n+1}(x, y)=3(y-1) H_{2, n} N_{n}^{2}+H_{2, n}^{3}+12 H_{2, n}^{2} N_{n}+3 H_{2, n}^{2} M_{n}+36 H_{2, n} N_{n}^{2}$

$$
\begin{aligned}
& +12 H_{2, n} N_{n} M_{n}+14 N_{n}^{3} \\
& +(x-1)\left((y-1) N_{n}^{3}+9 H_{2, n}^{2} N_{n}+6 H_{2, n}^{2} M_{n}+60 H_{2, n} N_{n}^{2}+42 H_{2, n} N_{n} M_{n}\right. \\
& \left.+3 H_{2, n} M_{n}^{2}+60 N_{n}^{3}+24 N_{n}^{2} M_{n}\right) \\
& +(x-1)^{2}\left(3 H_{2, n}^{2} M_{n}+27 H_{2, n} N_{n}^{2}+48 H_{2, n} N_{n} M_{n}+9 H_{2, n} M_{n}^{2}\right. \\
& \left.+72 N_{n}^{3}+75 N_{n}^{2} M_{n}+9 N_{n} M_{n}^{2}\right) \\
& +(x-1)^{3}\left(18 H_{2, n} N_{n} M_{n}+9 H_{2, n} M_{n}^{2}+27 N_{n}^{3}+78 N_{n}^{2} M_{n}+27 N_{n} M_{n}^{2}+M_{n}^{3}\right) \\
& +(x-1)^{4}\left(3 H_{2, n} M_{n}^{2}+27 N_{n}^{2} M_{n}+27 N_{n} M_{n}^{2}+3 M_{n}^{3}\right) \\
& +(x-1)^{5}\left(9 N_{n} M_{n}^{2}+3 M_{n}^{3}\right)+(x-1)^{6} M_{n}^{3},
\end{aligned}
$$


with initial conditions

$$
H_{2,1}(x, y)=y+2 \quad N_{1}(x, y)=M_{1}(x, y)=1 .
$$

As in Section 3, we will use these reduced formulas to compute several evaluations of the Tutte polynomial. Let us start by writing the reliability polynomial $R\left(\Sigma_{n}, p\right)$.

Proposition 4.7. For each $n \geq 1$, the reliability polynomial $R\left(\Sigma_{n}, p\right)$ is given by

$$
R\left(\Sigma_{n}, p\right)=p^{3^{n}-1}(1-p)^{\frac{3^{n}-1}{2}} H_{n}\left(1, \frac{1}{1-p}\right),
$$

with $H_{n}\left(1, \frac{1}{1-p}\right)=H_{2, n}\left(1, \frac{1}{1-p}\right)$ and

$$
\begin{gathered}
H_{2, n+1}\left(1, \frac{1}{1-p}\right)=\frac{p}{1-p} H_{2, n}^{3}+3 H_{2, n}^{3}+6 H_{2, n}^{2} N_{n} \\
N_{n+1}\left(1, \frac{1}{1-p}\right)=\frac{p}{1-p} H_{2, n}^{2} N_{n}+H_{2, n}^{3}+7 H_{2, n}^{2} N_{n}+H_{2, n}^{2} M_{n}+7 H_{2, n} N_{n}^{2} \\
M_{n+1}\left(1, \frac{1}{1-p}\right)=\frac{3 p}{1-p} H_{2, n} N_{n}^{2}+H_{2, n}^{3}+12 H_{2, n}^{2} N_{n}+3 H_{2, n}^{2} M_{n} \\
+36 H_{2, n} N_{n}^{2}+12 H_{2, n} N_{n} M_{n}+14 N_{n}^{3},
\end{gathered}
$$

with initial conditions

$$
H_{2,1}\left(1, \frac{1}{1-p}\right)=\frac{3-2 p}{1-p} \quad N_{1}\left(1, \frac{1}{1-p}\right)=M_{1}\left(1, \frac{1}{1-p}\right)=1 .
$$

Proof. By Lemma 4.6, one has $H_{1, n}(1, y)=H_{0, n}(1, y)=0$, for every $y \in \mathbb{R}$. Therefore, $H_{n}\left(1, \frac{1}{1-p}\right)=H_{2, n}\left(1, \frac{1}{1-p}\right)$; then it is enough to apply (1) of Theorem 2.6 and use Equations (18), (19) and (20).

Proposition 4.8. The complexity $\tau\left(\Sigma_{n}\right)$ is $H_{n}(1,1)=H_{2, n}(1,1)$, where

$$
\begin{gathered}
H_{2, n+1}(1,1)=3 H_{2, n}^{3}+6 H_{2, n}^{2} N_{n} \\
N_{n+1}(1,1)=H_{2, n}^{3}+7 H_{2, n}^{2} N_{n}+H_{2, n}^{2} M_{n}+7 H_{2, n} N_{n}^{2} \\
M_{n+1}(1,1)=H_{2, n}^{3}+12 H_{2, n}^{2} N_{n}+3 H_{2, n}^{2} M_{n}+36 H_{2, n} N_{n}^{2}+12 H_{2, n} N_{n} M_{n}+14 N_{n}^{3},
\end{gathered}
$$

with initial conditions

$$
H_{2,1}(1,1)=3 \quad N_{1}(1,1)=M_{1}(1,1)=1 .
$$

Proof. The complexity of $\Sigma_{n}$ is obtained by evaluating $H_{n}\left(1, \frac{1}{1-p}\right)=H_{2, n}\left(1, \frac{1}{1-p}\right)$ in $p=0$, using Equations (21), (22) and (23).

Remark 4.9. These formulas coincide with the relations obtained in [13, without using Tutte polynomials. More precisely, one can find in [13, Proposition 3.4]:

(1) $H_{n}(1,1)=\tau\left(\Sigma_{n}\right)=3^{\frac{3^{n}+2 n-1}{4}} 5^{\frac{3^{n}-2 n-1}{4}}$;

(2) $N_{n}(1,1)=3^{\frac{3^{n}-2 n-1}{4}} 5^{\frac{3^{n}-2 n-1}{4}} \cdot \frac{5^{n}-3^{n}}{2}$; 
(3) $M_{n}(1,1)=3^{\frac{3^{n}-6 n+3}{4}} 5^{\frac{3^{n}-2 n-1}{4}} \cdot\left(\frac{5^{n}-3^{n}}{2}\right)^{2}$.

Then, the asymptotic growth constant of the spanning trees of $\Sigma_{n}$ is

$$
\lim _{n \rightarrow \infty} \frac{\log \left(\tau\left(\Sigma_{n}\right)\right)}{\left|V\left(\Sigma_{n}\right)\right|}=\frac{1}{4}(\log 3+\log 5) .
$$

Evaluating $H_{n}\left(1, \frac{1}{1-p}\right)$ in $p=\frac{1}{2}$ gives the number of connected spanning subgraphs of $\Sigma_{n}$.

Proposition 4.10. The number of connected spanning subgraphs of $\Sigma_{n}$ is given by $H_{n}(1,2)=$ $H_{2, n}(1,2)$, with

$$
\begin{gathered}
H_{2, n+1}(1,2)=4 H_{2, n}^{3}+6 H_{2, n}^{2} N_{n} \\
N_{n+1}(1,2)=H_{2, n}^{3}+8 H_{2, n}^{2} N_{n}+H_{2, n}^{2} M_{n}+7 H_{2, n} N_{n}^{2} \\
M_{n+1}(1,2)=H_{2, n}^{3}+12 H_{2, n}^{2} N_{n}+3 H_{2, n}^{2} M_{n}+39 H_{2, n} N_{n}^{2}+12 H_{2, n} N_{n} M_{n}+14 N_{n}^{3},
\end{gathered}
$$

with initial conditions

$$
H_{2,1}(1,2)=4 \quad N_{1}(1,2)=M_{1}(1,2)=1 .
$$

Proof. One has $H_{n}(1,2)=H_{2, n}(1,2)$ since $H_{1, n}(1, y)=H_{0, n}(1, y)=0$, for every $y \in \mathbb{R}$ (see Lemma 4.6). Then it suffices to use Formula (2) of Theorem 2.5.

The following proposition about the number of spanning forests of $\Sigma_{n}$ holds.

Proposition 4.11. The number of spanning forests of $\Sigma_{n}$ is given by

$$
H_{n}(2,1)=H_{2, n}(2,1)+3 N_{n}(2,1)+M_{n}(2,1),
$$

where

$$
\begin{gathered}
H_{2, n+1}(2,1)=3 H_{2, n}^{3}+12 H_{2, n}^{2} N_{n}+6 H_{2, n} N_{n}^{2} \\
N_{n+1}(2,1)=H_{2, n}^{3}+12 H_{2, n}^{2} N_{n}+4 H_{2, n}^{2} M_{n}+28 H_{2, n} N_{n}^{2}+8 H_{2, n} N_{n} M_{n}+14 N_{n}^{3}+4 N_{n}^{2} M_{n} \\
M_{n+1}(2,1)=H_{2, n}^{3}+21 H_{2, n}^{2} N_{n}+12 H_{2, n}^{2} M_{n}+123 H_{2, n} N_{n}^{2}+120 H_{2, n} N_{n} M_{n}+24 H_{2, n} M_{n}^{2} \\
+173 N_{n}^{3}+204 N_{n}^{2} M_{n}+72 N_{n} M_{n}^{2}+8 M_{n}^{3}
\end{gathered}
$$

with initial conditions

$$
H_{2,1}(2,1)=3 \quad N_{1}(2,1)=M_{1}(2,1)=1 .
$$

Proof. It suffices to apply Formula (3) of Theorem 2.5] and to observe that $H_{1, n}(2, y)=N_{n}(2, y)$ and $H_{0, n}(2, y)=M_{n}(2, y)$, for each $y \in \mathbb{R}$.

Similarly to the case of Sierpiński graphs, the following proposition holds.

Proposition 4.12. $H_{n}(2,2)=2^{\left|E\left(\Sigma_{n}\right)\right|}=2^{\frac{3^{n+1}-3}{2}}$.

Proof. The proof is by induction. For $n=1$, we have $H_{1}(2,2)=8=2^{3}=2^{\left|E\left(\Sigma_{1}\right)\right|}$. Then, we recall that $\left|E\left(\Sigma_{n+1}\right)\right|=3\left|E\left(\Sigma_{n}\right)\right|+3$. An easy computation shows that $H_{n+1}(2,2)=8 H_{n}(2,2)^{3}$; therefore, $H_{n+1}(2,2)=8 H_{n}(2,2)^{3}=\left(2 \cdot 2^{\left|E\left(\Sigma_{n}\right)\right|}\right)^{3}=2^{3+3\left|E\left(\Sigma_{n}\right)\right|}=2^{\left|E\left(\Sigma_{n+1}\right)\right|}$. 
As regards the number of acyclic orientations of $\Sigma_{n}$, we have the following result.

Proposition 4.13. The number of acyclic orientations on $\Sigma_{n}$ is given by $H_{n}(2,0)$, where

$$
H_{n+1}(2,0)=\left(2 H_{n}(2,0)\right)^{3}-2\left(H_{2, n}(2,0)+N_{n}(2,0)\right)^{3}
$$

and

$$
\begin{gathered}
H_{2, n+1}(2,0)=2 H_{2, n}^{3}+12 H_{2, n}^{2} N_{n}+6 H_{2, n} N_{n}^{2} \\
N_{n+1}(2,0)=H_{2, n}^{3}+11 H_{2, n}^{2} N_{n}+4 H_{2, n}^{2} M_{n}+28 H_{2, n} N_{n}^{2}+8 H_{2, n} N_{n} M_{n}+14 N_{n}^{3}+4 N_{n}^{2} M_{n} \\
M_{n+1}(2,0)=H_{2, n}^{3}+21 H_{2, n}^{2} N_{n}+12 H_{2, n}^{2} M_{n}+120 H_{2, n} N_{n}^{2}+120 H_{2, n} N_{n} M_{n}+24 H_{2, n} M_{n}^{2} \\
+172 N_{n}^{3}+204 N_{n}^{2} M_{n}+72 N_{n} M_{n}^{2}+8 M_{n}^{3},
\end{gathered}
$$

with initial conditions

$$
H_{2,1}(2,0)=2 \quad N_{1}(2,0)=M_{1}(2,0)=1 .
$$

Proof. It suffices to apply Formula (5) of Theorem 2.5. Then, one can directly verify that the Tutte polynomial $H_{2, n+1}(2,0)+3 H_{1, n+1}(2,0)+H_{0, n+1}(2,0)$ can be rewritten as $\left(2 H_{n}(2,0)\right)^{3}-$ $2\left(H_{2, n}(2,0)+N_{n}(2,0)\right)^{3}$.

Next, let us look at the chromatic polynomial of $\Sigma_{n}$.

Proposition 4.14. For each $n \geq 1$, the chromatic polynomial $\chi_{n}(\lambda)$ of the Schreier graph $\Sigma_{n}$ is

$$
\chi_{n}(\lambda)=(-1)^{3^{n}-1} \lambda P_{n}(\lambda),
$$

where $P_{n}(\lambda)=P_{2, n}(\lambda)+3 P_{1, n}(\lambda)+P_{0, n}(\lambda)$, with $P_{i, n}(\lambda)=H_{i, n}(1-\lambda, 0)$, for each $i=1,2,3$.

Proof. It is an easy consequence of Equation (2) of Theorem 2.6. Here we omit the explicit recursive equations of $P_{i, n}(\lambda)$.

Also in the case of the Schreier graph $\Sigma_{n}$, we can explicitly study the relationship between the evaluation of the Tutte polynomial on the hyperbola $(x-1)(y-1)=2$ and the partition function of the Ising model. In [14, Theorem 3.3], the partition function of the Ising model on $\Sigma_{n}$ has been described as

$$
Z_{n}=2^{3^{n}} \cosh (\beta J)^{\frac{3^{n+1}-3}{2}} \Psi_{n}(\tanh (\beta J)),
$$

with

$$
\Psi_{n}(z)=z^{3^{n}} \prod_{k=1}^{n} \psi_{k}^{3^{n-k}}(z)\left(\psi_{n+1}(z)-1\right),
$$

where $\psi_{1}(z)=\frac{z+1}{z}$ and $\psi_{k}(z)=\psi_{k-1}^{2}(z)-3 \psi_{k-1}(z)+4$, for each $k \geq 2$.

Theorem 4.15. For each $n \geq 1$, one has

$$
2\left(e^{2 \beta J}-1\right)^{\left|V\left(\Sigma_{n}\right)\right|-1} e^{-\beta J\left|E\left(\Sigma_{n}\right)\right|} H_{n}\left(\frac{e^{2 \beta J}+1}{e^{2 \beta J}-1}, e^{2 \beta J}\right)=Z_{n} .
$$


Proof. The proof can be done by induction and follows the same strategy as for the Sierpiński graphs (see Theorem 3.17). Recall that $\left|E\left(\Sigma_{n}\right)\right|=\frac{3^{n+1}-3}{2}$ and $\left|V\left(\Sigma_{n}\right)\right|=3^{n}$. Putting, as usual, $e^{\beta J}=t$, Equation (24) can be written as

$$
\frac{2\left(t^{2}-1\right)^{3^{n}-1}}{t^{\frac{3^{n+1}-3}{2}}} H_{n}\left(\frac{t^{2}+1}{t^{2}-1}, t^{2}\right)=2^{3^{n}}\left(\frac{t^{2}+1}{2 t}\right)^{\frac{3^{n+1}-3}{2}} \Psi_{n}\left(\frac{t^{2}-1}{t^{2}+1}\right)
$$

or, more explicitly,

$$
\frac{2\left(t^{2}-1\right)^{3^{n}-1}}{t^{\frac{3^{n+1}-3}{2}}} H_{n}\left(\frac{t^{2}+1}{t^{2}-1}, t^{2}\right)=\left.2^{3^{n}}\left(\frac{t^{2}+1}{2 t}\right)^{\frac{3^{n+1}-3}{2}}\left(\frac{t^{2}-1}{t^{2}+1}\right)^{3^{n}} \prod_{k=1}^{n} \psi_{k}^{3^{n-k}}(z)\left(\psi_{n+1}(z)-1\right)\right|_{z=\frac{t^{2}-1}{t^{2}+1}} .
$$

For each $n \geq 1$, set

$$
A_{n}^{\prime}(x, y)=H_{2, n}(x, y)+H_{1, n}(x, y) \quad B_{n}^{\prime}(x, y)=2 H_{1, n}(x, y)+H_{0, n}(x, y)
$$

and

$$
C_{n}^{\prime}=\frac{2\left(t^{2}-1\right)^{3^{n}-1}}{t^{\frac{3^{n+1}-3}{2}}} \quad D_{n}^{\prime}=2^{3^{n}}\left(\frac{t^{2}+1}{2 t}\right)^{\frac{3^{n+1}-3}{2}}\left(\frac{t^{2}-1}{t^{2}+1}\right)^{3^{n}-1}
$$

Since $H_{n}(x, y)=A_{n}^{\prime}(x, y)+B_{n}^{\prime}(x, y)$, it is enough to prove the equations

$$
C_{n}^{\prime} A_{n}^{\prime}\left(\frac{t^{2}+1}{t^{2}-1}, t^{2}\right)=\left.D_{n}^{\prime} \prod_{k=1}^{n} \psi_{k}^{3^{n-k}}(z)\right|_{z=\frac{t^{2}-1}{t^{2}+1}}
$$

and

$$
C_{n}^{\prime} B_{n}^{\prime}\left(\frac{t^{2}+1}{t^{2}-1}, t^{2}\right)=\left.D_{n}^{\prime} \prod_{k=1}^{n} \psi_{k}^{3^{n-k}}(z)\left(z\left(\psi_{n+1}(z)-1\right)-1\right)\right|_{z=\frac{t^{2}-1}{t^{2}+1}} .
$$

They can be proven by induction, using the following relations obtained by the evaluations of Equations (14), (15) and (16) on the hyperbola $(x-1)(y-1)=2$ :

$$
\begin{gathered}
A_{n+1}^{\prime}=\frac{(y+1) A_{n}^{\prime 2}\left(B_{n}^{\prime}+y B_{n}^{\prime}+2 y A_{n}^{\prime}\right)}{2(y-1)} \\
B_{n+1}^{\prime}=\frac{\left(B_{n}^{\prime}+y B_{n}^{\prime}+2 y A_{n}^{\prime}\right)\left(4 y A_{n}^{\prime} B_{n}^{\prime}+y^{2} A_{n}^{\prime} B_{n}^{\prime}+y^{2} B_{n}^{\prime 2}+3 A_{n}^{\prime} B_{n}^{\prime}+2 y B_{n}^{\prime 2}+4 A_{n}^{\prime 2}+B_{n}^{\prime 2}\right)}{2(y-1)^{2}},
\end{gathered}
$$

with initial conditions

$$
A_{1}^{\prime}\left(\frac{y+1}{y-1}, y\right)=\frac{y(y+1)}{y-1} \quad B_{1}^{\prime}\left(\frac{y+1}{y-1}, y\right)=\frac{4 y}{(y-1)^{2}} .
$$

\section{Acknowledgements}

We wish to express our deepest gratitude to Tullio Ceccherini-Silberstein and Toni Machì for useful comments and suggestions. We are very grateful to the referee for several remarks which improved the presentation of the paper. 


\section{REFERENCES}

[1] P. D. Alvarez, F. Canfora and L. Parisi, Partition function of the Potts model on self-similar lattices as a dynamical system and multiple transitions, preprint, available at http://arxiv.org/abs/1007.4082

[2] L. Bartholdi, R. Grigorchuk and V. Nekrashevych, From fractal groups to fractal sets, in Fractals in Graz, eds. P. Grabner and W. Woess, Trends in Mathematics, Birkäuser Verlag, Basel, 2003, 25-118.

[3] L. Bartholdi and B. Virág, Amenability via random walks, Duke Math. J., 130 (1) (2005), 39-56.

[4] N. L. Biggs, R. M. Damerell and D. A. Sands, Recursive Families of Graphs, J. Combinatorial Theory, 12 (B) (1972), 123-131.

[5] B. Bollobás, Modern Graph Theory, Graduate Texts in Mathematics, 184, Springer-Verlag, New York (1998).

[6] T. Brylawski and J. Oxley, The Tutte polynomial and its Applications, in Matroid Applications, ed. N. White, Encyclopedia Math. Appl., 40, Cambridge Univ. Press, Cambridge, 1992, 123-225.

[7] T. Ceccherini-Silberstein, A. Donno and D. Iacono, The Tutte Polynomial of the Schreier graphs of the Grigorchuk group and the Basilica group, in Ischia Group Theory 2010: Proceedings of the Conference (M. Bianchi, P. Longobardi, M. Maj and C. M. Scoppola editors), 45-68. World Scientific, Singapore, Toh Tuck Link (2011).

[8] S.-C. Chang, Acyclic orientations on the Sierpiński gasket, available at http://arxiv.org/abs/1005.3627v1

[9] S.-C. Chang and L.-C. Chen, Spanning forests on the Sierpiński Gasket, Discrete Math. Theor. Comput. Sci., 10 (2) (2008), 55-76.

[10] S.-C. Chang and L.-C. Chen, Number of connected spanning subgraphs on the Sierpinski Gasket, Discrete Math. Theor. Comput. Sci., 11 (1) (2009), 55-77.

[11] S.-C. Chang, L.-C. Chen and W.-S. Yang, Spanning Trees on the Sierpiński Gasket, J. Stat. Phys., 126 (3) (2007), 649-667.

[12] R. Cori and Y. Le Borgne, The sand-pile model and Tutte polynomials. Formal power series and algebraic combinatorics, (Scottsdale, AZ, 2001), Adv. in Appl. Math., 30, Issue 1-2, (2003), 44-52.

[13] D. D'Angeli and A. Donno, Weighted spanning trees on some self-similar graphs, Electron. J. Combin., Vol. 18 (1), P16 (28 pages), 2011.

[14] D. D'Angeli, A. Donno and T. Nagnibeda, Partition functions of the Ising model on some self-similar Schreier graphs, in Progress in Probability: Random Walks, Boundaries and Spectra, D.Lenz, F. Sobieczky and W. Woess editors, 64 (2011), 277-304, Springer Basel.

[15] D. D'Angeli, A. Donno and T. Nagnibeda, Counting dimer coverings on self-similar Schreier graphs, European J. Combin., 33 (7) (2012), 1484-1513.

[16] J.A. Ellis-Monaghan and C. Merino, Graph Polynomials and Their Applications I: The Tutte Polynomial, in Structural Analysis of Complex Networks, ed. M. Dehmer, (2011), 219-255.

[17] R. Grigorchuk, Solved and unsolved problems around one group, in Infinite groups: geometric, combinatorial and dynamical aspects, eds. L. Bartholdi, T. Ceccherini-Silberstein, T. Smirnova-Nagnibeda and A. Żuk, Progr. Math., 248, Birkhäuser, Basel, 2005, 117-218.

[18] R. Grigorchuk and Z. Šunić, Self-similarity and branching in group theory, in Groups St. Andrews 2005, I, London Math. Soc. Lecture Note Ser., 339, Cambridge Univ. Press, Cambridge, 2007, 36-95.

[19] R. Grigorchuk and A. Żuk, On a torsion-free weakly branch group defined by a three-state automaton, Internat. J. Algebra Comput., 12 (1-2) (2002), 223-246.

[20] S. Klavžar, Coloring Sierpiński graphs and Sierpiński gasket graphs, Taiwanese J. Math., 12 (2) (2008), 513-522.

[21] R. Lyons, Asymptotic enumeration of spanning trees, Combin. Probab. Comput., 14 (4) (2005), 491-522.

[22] C. Merino, Chip-firing and the Tutte polynomial, Ann. Comb., 1 (3) (1997), 253-259.

[23] V. Nekrashevych, Self-similar Groups, Mathematical Surveys and Monographs, 117, American Mathematical Society, Providence, RI, 2005.

[24] M. Noy and A. Ribó, Recursively constructible families of graphs, Adv. in Appl. Math., 32 (2004), 350-363.

[25] J. Oxley and D.J.A. Welsh, The Tutte polynomial and percolation, in Graph theory and related topics, Proc. Conf., Univ. Waterloo, Waterloo, Ont., 1977, eds. J.A. Bondy and U.S.R. Murty, Academic Press, New York-London, 1979, 329-339.

[26] E. Teufl and S. Wagner, The number of spanning trees of finite Sierpiński graphs, Proceedings of the Fourth Colloquium on Mathematics and Computer Science, Nancy, September 18-22, 2006, 411-414.

[27] E. Teufl and S. Wagner, Enumeration of matchings in families of self-similar graphs, Discrete Appl. Math., 158 (14), (2010), 1524-1535

[28] W.T. Tutte, A ring in graph theory, Proc. Cambridge Philos. Soc., 43, (1947), 26-40.

[29] W.T. Tutte, A contribution to the theory of chromatic polynomials, Canadian. J. Math., 6, (1954), 80-91. 
[30] W.T. Tutte, On dichromatic polynomials, J. Combinatorial Theory, 2, (1967), 301-320.

[31] W.T. Tutte, Graph-polynomials, Special issue on the Tutte polynomial, Adv. in Appl. Math., 32 (1-2) (2004), 5-9.

[32] D.J.A. Welsh and C. Merino, The Potts model and the Tutte polynomial. Probabilistic techniques in equilibrium and nonequilibrium statistical physics, J. Math. Phys., 41 (3) (2000), 1127-1152. 